Noname manuscript No.

(will be inserted by the editor)

\title{
Guiding dose selection of monoclonal antibodies using a new parameter (AFTIR) for characterizing ligand binding systems
}

\author{
Sameed Ahmed • Miandra Ellis . \\ Hongshan Li · Luca Pallucchini . \\ Andrew M. Stein
}

Sameed, Miandra, Hongshan, and Luca all contributed equally to this manuscript.

Keywords monoclonal antibody - target mediated drug disposition · pharmacokinetics and pharmacodynamics $\cdot$ pharmacometrics

Sameed Ahmed

Department of Applied Mathematics, University of Waterloo

E-mail: sameed.ahmed@uwaterloo.ca

Miandra Ellis

School of Mathematical and Statistical Sciences, Arizona State University

E-mail: mellis5@asu.edu

Hongshan $\mathrm{Li}$

Department of Mathematics, Purdue University

E-mail: li108@purdue.edu

Luca Pallucchini

Department of Mathematics, Temple University

E-mail: luca.pallucchini@temple.edu

Andrew M. Stein

Novartis Institute for BioMedical Research, 45 Sidney St, Cambridge MA, 02140 USA

E-mail: andrew.stein@novartis.com 


\begin{abstract}
Guiding the dose selection for monoclonal antibody oncology drugs is often done using methods for predicting the receptor occupancy of the drug in the tumor. In this manuscript, previous work on characterizing target inhibition at steady state using the AFIR metric [1] is extended to include a "target-tissue" compartment and the shedding of membrane-bound targets. A new potency metric AFTIR (Averarge Free Tissue target to Initial target ratio at steady state) is derived, and it depends on only four key quantities: the equilibrium binding constant, the fold-change in target expression at steady state after binding to drug, the biodistribution of target from circulation to target tissue, and the average drug concentration in circulation. The AFTIR metric is useful for guiding dose selection, for efficiently performing sensitivity analyses, and for building intuition for more complex target mediated drug disposition models. In particular, reducing the complex, physiological model to four key parameters needed to predict target inhibition helps to highlight specific parameters that are the most important to estimate in future experiments to guide drug development.
\end{abstract}




\section{Introduction}

During biologic drug development, prediction of target engagement at the site of action plays a critical role in dose regimen selection [2]. Because target engagement measurements at the site of action are often impossible to obtain, model-based predictions of target engagement at the site of action are often used to help justify the dose regimen selection. The methods used to predict target engagement vary significantly in their assumptions and in their level of complexity. For example, consider the model-based dose selection of pembrolizumab and atezolizumab.

For the PD-1 inhibitor pembrolizumab, a physiologically based model for antibody distribution and target engagement was developed to predict the dose needed to achieve target engagement and tumor suppression [3]. This model involved many assumptions about a large number of parameters and how these parameters scale from mice to humans. For the PD-L1 inhibitor atezolizumab, a much simpler approach was taken to guide the dosing regimen [4]. The tumor biodistribution coefficient $(B)$ and in vivo binding affinity $\left(K_{\mathrm{d}}\right)$ were chosen based on preclinical data. The steady state trough concentration $\left(C_{\text {trough }}\right)$ was estimated from clinical observations. The receptor occupancy $(R O)$ formula in Equation 1 was then used to identify the dosing regimen needed to achieve 95\% target occupancy. This approach made fewer assumptions about model parameters than in 3. However, in choosing this simple model, many implicit assumptions were made about the system. For example, this simple model assumes that the rate of PD-L1 internalization does not change when it is bound to atezolizumab.

$$
R O=B \cdot C_{\text {trough }} /\left(B \cdot C_{\text {trough }}+K_{\mathrm{d}}\right)
$$

The complex, mechanistic model used for pembrolizumab, captures more details of the underlying physiological processes. However, this model was more difficult to analyze due to it's complexity; it also had a large number of unknown parameters which were difficult to estimate accurately. The simple model used for atezolizumab was easier to analyize and had fewer unknown parameters to estimate. However, this model required certain implicit assumptions which do not necessarily hold.

In this paper, a mathematical analysis of a physiologically-based model for drug distribution and target turnover is performed to derive a simple potency factor for characterizing target engagement. This theoretical calculation is validated using simulations of the model. All assumptions made in deriving this formula are explicitly stated. This paper extends previous work that focused on target engagement in circulation, as characterized by the Average Free target to Initial target Ratio (AFIR) at steady state in circulation [1]. 


\section{Theory}

In this section, an expression for the Average Free Tissue target to Initial target Ratio in tissue (AFTIR) is derived for the model in Figure 1

\section{Model Description}

The model in Figure 1 is based on the standard target mediated drug dispositon (TMDD) model [5], where a drug $(D)$ binds its target. The model is extended to include the following processes:

- Shedding of the membrane-bound target $(M)$ to form soluble target $(S)$.

- Binding of the drug to both the membrane-bound target and the soluble target to form complexes $D M$ and $D S$, respectively.

- Distribution of drug, target, and complexes from central (1) to tissue (3) compartment via either passive processes (soluble target) or active processes such as cell-trafficking (membrane-bound target).

As with the standard TMDD model, the peripheral compartment (2) contains the drug only; it is included so that the two-compartment pharmacokinetics of the drug can be described. The ordinary differential equations for describing this system are given by Equations (2) - (12). 


$$
\begin{aligned}
& \frac{d D_{1}}{d t}=\frac{1}{V_{C}} \operatorname{Dose}_{i v}(t)-k_{12 D} D_{1}+\frac{V_{D_{2}}}{V_{D_{1}}} k_{21 D} D_{2}-k_{13 D} D_{1} \\
& +\frac{V_{D_{3}}}{V_{D_{1}}} k_{31 D} D_{3}-k_{e D 1} D_{1}-k_{\mathrm{on} 1} D_{1} \cdot S_{1}+k_{\mathrm{off} 1}\left(D S_{1}\right) \\
& -k_{\mathrm{on} 1} D_{1} \cdot M_{1}+k_{\mathrm{off} 1}\left(D M_{1}\right) \\
& \frac{d D_{2}}{d t}=k_{12 D} \frac{V_{D_{1}}}{V_{D_{2}}} D_{1}-k_{21 D} D_{2} \\
& \frac{d D_{3}}{d t}=\frac{V_{D_{1}}}{V_{D_{3}}} k_{13 D} D_{1}-k_{31 D} D_{3}-k_{e D 3} D_{3}-k_{\mathrm{on} 3} D_{3} \cdot M_{3}+k_{\mathrm{off} 3}\left(D M_{3}\right) \\
& -k_{\mathrm{on} 3} D_{3} \cdot S_{3}+k_{\mathrm{off} 3}\left(D S_{3}\right) \\
& \frac{d M_{1}}{d t}=k_{\mathrm{syn} M 1}-k_{\mathrm{shed} M_{1}} M_{1}-k_{13 M} M_{1}+\frac{V_{M_{3}}}{V_{M_{1}}} k_{31 M} M_{3}-k_{e M 1} M_{1} \\
& -k_{\mathrm{on} 1} D_{1} \cdot M_{1}+k_{\mathrm{off} 1}\left(D M_{1}\right) \\
& \frac{d M_{3}}{d t}=k_{\mathrm{syn} M 3}-k_{\mathrm{shed} M_{3}} M_{3}+\frac{V_{M_{1}}}{V_{M_{3}}} k_{13 M} M_{1}-k_{31 M} M_{3}-k_{e M 3} M_{3} \\
& -k_{\mathrm{on} 3} D_{3} \cdot M_{3}+k_{\mathrm{off} 3}\left(D M_{3}\right) \\
& \frac{d S_{1}}{d t}=k_{\mathrm{syn} S 1}+k_{\mathrm{shed} M_{1}} M_{1}-k_{13 S} S_{1}+\frac{V_{S_{3}}}{V_{S_{1}}} k_{31 S} S_{3}-k_{e S 1} S_{1} \\
& -k_{\mathrm{on} 1} D_{1} \cdot S_{1}+k_{\mathrm{off} 1}\left(D S_{1}\right) \\
& \frac{d S_{3}}{d t}=k_{\mathrm{syn} S 3}+k_{\mathrm{shed} M_{3}} M_{3}+\frac{V_{S_{1}}}{V_{S_{3}}} k_{13 S} S_{1}-k_{31 S} S_{3}-k_{e S 3} S_{3} \\
& -k_{\mathrm{on} 3} D_{3} \cdot S_{3}+k_{\mathrm{off3}}\left(D S_{3}\right) \\
& \frac{d\left(D M_{1}\right)}{d t}=-k_{\text {shed } D M_{1}} D M_{1}-k_{13 D M}\left(D M_{1}\right)+\frac{V_{D M_{3}}}{V_{D M_{1}}} k_{31 D M}\left(D M_{3}\right) \\
& -k_{e D M 1} D M_{1}+k_{\mathrm{on} 1} D_{1} \cdot M_{1}-k_{\mathrm{off} 1}\left(D M_{1}\right) \\
& \frac{d\left(D M_{3}\right)}{d t}=-k_{\text {shed } D M_{3}} D M_{3}+\frac{V_{D M_{1}}}{V_{D M_{3}}} k_{13 D M}\left(D M_{1}\right)-k_{31 D M}\left(D M_{3}\right) \\
& -k_{e D M} D M_{3}+k_{\mathrm{on} 3} D_{3} \cdot M_{3}-k_{\mathrm{off} 3}\left(D M_{3}\right) \\
& \frac{d\left(D S_{1}\right)}{d t}=k_{\text {shed } D M_{1}} D M_{1}-k_{13 D S} D S_{1}+\frac{V_{D S_{3}}}{V_{D S_{1}}} k_{31 D S} D S_{3} \\
& -k_{e D S 1}\left(D S_{1}\right)+k_{\mathrm{on} 1} D_{1} \cdot S_{1}-k_{\mathrm{off} 1}\left(D S_{1}\right) \\
& \frac{d\left(D S_{3}\right)}{d t}=k_{\text {shed } D M_{3}} D M_{3}+\frac{V_{D S_{1}}}{V_{D S_{3}}} k_{13 D S} D S_{1}-k_{31 D S} D S_{3} \\
& -k_{e D S 3}\left(D S_{3}\right)+k_{\mathrm{on} 3} D_{1} \cdot S_{3}-k_{\mathrm{off} 1}\left(D S_{3}\right)
\end{aligned}
$$

The initial conditions for all variables are zero, except for the free target concentrations $M_{1}, M_{3}, S_{1}$, and $S_{3}$. The equations used to determine these four initial concentrations are provided in Appendix A.1.1. 
Checking for tumor homogeneity using the Thiele Modulus

In setting up the compartmental model in Figure 1, an implicit assumption is made that all compartments can be treated as homogeneous, well-mixed tissues. This assumption is reasonable for the circulating compartment, and it is not needed for the peripheral compartment, which is an empirical model feature that is used to get the standard 2-compartment PK. But when the target is membrane-bound, then for the tissue compartment, this assumption will not be correct for low doses where the drug may be internalized and eliminated before it penetrates through the tissue. For membrane-bound targets, the assumption of a homogeneous, well-mixed tissue compartment can be evaluated by checking whether the Thiele modulus given below is less than one. 6.7.

$$
\phi_{M}^{2}=\frac{\text { Drug Elimination from Tumor }}{\text { Drug Penetration into Tumor }}=\frac{\left(k_{\mathrm{shed} D M 3}+k_{\mathrm{e} D 3}+k_{\mathrm{e} D M 3}\right) \cdot M_{3 \mathrm{tot}}}{k_{13 D} \cdot C_{\mathrm{avg}} \cdot\left(V_{D 1} / V_{D 3}\right)}
$$

The Thiele Modulus is a non-dimensional parameter which is the ratio of receptor elimination (due to shedding and internalization) to the drug penetration into the tissue. Here, $M_{3 \text { tot }}$ is the total concentration of receptors in the tissue at steady state, which is often assumed to be equal to $M_{30}$, but could also be set to $M_{30} \cdot T_{\text {fold }}$, where $T_{\text {fold }}$ is the fold-change in receptor expression at steady state and is discussed further below. $T_{\text {fold }}$ is derived in Appendix A.1. To our knowledge, it has not been established yet how much less than one the Thiele modulus must be for the assumption of tissue homogeneity to hold.

\section{AFTIR derivation}

\section{AFTIR definition}

Average Free Tissue target to Initial target Ratio (AFTIR) is defined as

$$
\text { AFTIR }:=\frac{1}{\tau} \int_{t_{\mathrm{ss}}}^{t_{\mathrm{ss}}+\tau} \frac{T_{3}(t)}{T_{30}} d t=\frac{T_{3 \mathrm{avg}, \mathrm{ss}}}{T_{30}}=\left(\frac{T_{3 \mathrm{avg}, \mathrm{ss}}}{T_{3 \mathrm{tot}, \mathrm{ss}}}\right)\left(\frac{T_{3 \mathrm{tot}, \mathrm{ss}}}{T_{30}}\right) .
$$

This equation applies for both membrane-bound $(M)$ and soluble $(S)$ targets, where $T_{3}(t)$ is the time series target concentration in the tumor compartment, $T_{30}$ is the baseline target, $T_{3 \mathrm{avg}, \mathrm{ss}}$ is the average free target concentration at steady state, and $T_{3 \text { tot,ss }}$ denotes the total target concentration at steady state. AFTIR gives a measure of target inhibition in the tissue of interest (compartment 3) where the lower the AFTIR value, the greater the inhibition. The objective of this section is to derive the following estimate for AFTIR:

$$
\text { AFTIR } \approx \frac{K_{\mathrm{eq}} \cdot T_{\mathrm{fold}}}{K_{\mathrm{eq}} \cdot T_{\mathrm{fold}}+B_{\mathrm{sat}}^{\mathrm{ISF}} \cdot C_{\mathrm{avg}}},
$$

where $K_{\text {eq }}$ is an equilibrium constant measuring how fast the drug and target bind to form complexs, $T_{\text {fold }}$ is the fold-change in the target levels at steady 
state compared to baseline, $B_{\text {sat }}^{\text {ISF }}$ is the biodistribution coefficient (i.e. the fraction of the drug from circulation that is found in the tissue of interest), and $C_{\text {avg }}$ is the average drug concentration in circulation. Expressions for each of these terms are derived below.

\section{$K_{\text {eq }}$ definition}

Three different equilibrium constants are defined below. (1) The quasi-equilibrium approximation for both membrane and soluble target is 8

$$
K_{\mathrm{d}}:=\frac{k_{\mathrm{off} 3}}{k_{\mathrm{on} 3}}
$$

(2) The quasi-steady state approximation for soluble targets is 8

$$
K_{\mathrm{ss}, \mathrm{sol}}:=\frac{k_{\mathrm{off} 3}+k_{\mathrm{e} D S 3}}{k_{\mathrm{on} 3}} .
$$

For membrane-bound targets, the formula is similar, though shedding needs to be taken into account as well:

$$
K_{\mathrm{ss}, \mathrm{mem}}:=\frac{k_{\mathrm{off} 3}+k_{\mathrm{e} D M 3}+k_{\mathrm{shed} D M 3}}{k_{\mathrm{on} 3}} .
$$

(3) A new equilibrium constant is introduced in this paper, called the quasisteady-state with distribution constant, denoted $K_{\mathrm{ssd}}$. This new equilibrium constant outperforms $K_{\mathrm{d}}$ and $K_{\mathrm{SS}}$ in terms of accuracy of approximating AFTIR.

The derivation of $K_{\text {ssd }}$ for membrane-bound targets is shown below, and a similar process is followed for the soluble target version. To begin, assume a constant infusion of drug and that the system has reached steady state. Setting Equation 10 to zero and rearranging terms gives

$$
\begin{aligned}
k_{\mathrm{on} 3} D_{3} \cdot M_{3} & =k_{\mathrm{shed} D M_{3}}\left(D M_{3}\right)+k_{31 D M}\left(D M_{3}\right)+k_{\mathrm{e} D M 3}\left(D M_{3}\right) \\
& +k_{\mathrm{off} 3}\left(D M_{3}\right)+\frac{V_{D M_{1}}}{V_{D M_{3}}} k_{13 D M}\left(D M_{1}\right)
\end{aligned}
$$

Dividing each side by $k_{\mathrm{on} 3} \cdot D M_{3}$ gives

$$
\begin{aligned}
\frac{D_{3} \cdot M_{3}}{D M_{3}} & =\frac{k_{\mathrm{shed} D M_{3}}+k_{31 D M}+k_{\mathrm{e} D M 3}+k_{\mathrm{off} 3}}{k_{\mathrm{on} 3}} \\
& +\frac{V_{D M_{1}}}{V_{D M_{3}}} \frac{D M_{1}}{D M_{3}} \frac{k_{13 D M}}{k_{\mathrm{on} 3}} .
\end{aligned}
$$

We then define $K_{\text {ssd }}$ (for membrane-bound target and soluble target) as

$$
\begin{aligned}
K_{\mathrm{ssd}, \mathrm{mem}} & :=\frac{k_{\mathrm{shed} D M_{3}}+k_{31 D M}+k_{\mathrm{e} D M 3}+k_{\mathrm{off} 3}}{k_{\mathrm{on} 3}}, \\
K_{\mathrm{ssd}, \mathrm{sol}} & :=\frac{k_{31 D S}+k_{\mathrm{e} D S 3}+k_{\mathrm{off} 3}}{k_{\mathrm{on} 3}} .
\end{aligned}
$$


Substituting Equation 21 into Equation gives

$$
\begin{aligned}
\frac{D_{3} \cdot M_{3}}{D M_{3}} & =K_{\mathrm{ssd}, \mathrm{mem}}+\frac{V_{D M_{1}}}{V_{D M_{3}}} \frac{D M_{1}}{D M_{3}} \frac{k_{13 D M}}{k_{\mathrm{on} 3}} \\
& =K_{\mathrm{ssd}, \mathrm{mem}}+\frac{\mathrm{Amt}_{D M 1}}{\operatorname{Amt}_{D M 3}} \frac{k_{13 D M}}{k_{\mathrm{on} 3}},
\end{aligned}
$$

where $\operatorname{Amt}_{D M 1}=V_{D M_{1}} \cdot D M_{1}$ is the amount of complex in the central compartment, and $\mathrm{Amt}_{D M 3}=V_{D M_{3}} \cdot D M_{3}$ is the amount of complex in the tissue compartment. If the membrane-bound target is unable to move to the central compartment (i.e., $k_{13 D M}=k_{31 D M}=0$ ), or if the amount of complex is much larger in the tissue than in circulation such that $\mathrm{Amt}_{D M 1} / \mathrm{Amt}_{D M 3} \approx 0$, then

$$
\frac{D_{3} \cdot M_{3}}{D M_{3}}=K_{\mathrm{ssd}, \mathrm{mem}}
$$

And similarly for soluble targets,

$$
\frac{D_{3} \cdot S_{3}}{D S_{3}}=K_{\mathrm{ssd}, \mathrm{sol}}
$$

Regardless of which $K_{\text {eq }}$ is used, the following approximation is assumed to hold:

$$
K_{\mathrm{eq}}=\frac{D_{3} \cdot T_{3}}{D T_{3}}
$$

$T_{\text {fold }}, B_{\text {sat }}^{I S F}$, and $C_{\text {avg }}$ definition

The total target in tissue is defined as $T_{3 \text { tot }}:=T_{3}+D T_{3}$. The fold-accumulation of the target in tissue at steady state is defined as

$$
T_{\text {fold }}:=\frac{T_{3 \text { tot, ss }}}{T_{30}} .
$$

The analytical formula for $T_{\text {fold }}$ is derived in Appendix A.1 for the scenario where there is shedding of membrane-bound target to form soluble target. The scenario where no shedding takes place is calculated by setting either all shedding rates $\left(k_{\text {shed }}\right)$ terms to zero (so that there is no shedding), or, for soluble targets, by setting all membrane-bound target synthesis rates $\left(k_{\mathrm{syn} M}\right)$ to zero.

For large drug concentrations that saturate the target, the steady state biodistribution coefficient $\left(B_{\mathrm{sat}}^{\mathrm{ISF}}\right)$ gives the the ratio of the drug concentration in tissue interstitial fluid (ISF) to that in circulation. The average steady state circulating concentration is defined as $C_{\text {avg }}:=D_{1 \mathrm{avg}, \mathrm{ss}}$. The analytical formula for $B_{\mathrm{sat}}^{\mathrm{ISF}}$ is derived in Appendix $\mathrm{A.2}$ and is in Equation 27.

$$
B_{\mathrm{sat}}^{\mathrm{ISF}}:=\frac{D_{3 \mathrm{avg}, \mathrm{ss}}}{D_{1 \mathrm{avg}, \mathrm{ss}}}=\frac{D_{3 \mathrm{avg}, \mathrm{ss}}}{C_{\mathrm{avg}}}=\frac{V_{D 1}}{V_{D 3}} \cdot \frac{k_{13 D}}{\left(k_{\mathrm{e} D 3}+k_{31 D}\right)} .
$$

The above formula only applies to large doses that saturate the target. For low doses that do not saturate the target, binding to the target can significantly 
impact amount of drug that distributes to the tissue. Besides $B_{\text {sat }}^{\text {ISF }}$, there are two other biodistribution terms that are also referenced in the literature: $B_{\text {free }}\left(C_{\text {avg }}, T, K_{\text {eq }}\right)$ and $B_{\text {tot }}\left(C_{\text {avg }}, T, K_{\text {eq }}\right)$, which refer to the fraction of free drug and total drug that is found in tissue compared to circulation. Both $B_{\text {free }}$ and $B_{\text {tot }}$ depend on the circulating drug concentration, level of target expression, and binding affinity. Throughout this manuscript, it is $B_{\text {sat }}^{\text {ISF }}$ that will be used.

\section{Putting it all together}

Using the definitions of $T_{3 \text { tot }}$ and $K_{\text {eq }}$, substituting the equation $D T_{3}=T_{3 \text { tot }}$ $T_{3}$ into the equation $K_{\text {eq }}=D \cdot T /(D T)$, and solving for $T_{3} / T_{3 \text { tot }}$ gives

$$
\frac{T_{3}}{T_{3 \mathrm{tot}}}=\frac{K_{\mathrm{eq}}}{K_{\mathrm{eq}}+D_{3}} \approx \frac{K_{\mathrm{eq}}}{D_{3}} \approx \frac{K_{\mathrm{eq}}}{D_{3 \mathrm{tot}}} .
$$

The first approximation holds when $D_{3} \gg K_{\text {eq }}$, and the second approximation holds when $D_{3 \text { tot }} \approx D_{3}$, which occurs when drug is dosed in vast molar excess to the target, as is the case for most monoclonal antibody (mAb) drugs at the approved dosing regimen.

Using Equations (26) and 28) gives

$$
\frac{T_{3}}{T_{30}}=\left(\frac{T_{3}}{T_{3 \mathrm{tot}}}\right)\left(\frac{T_{3 \mathrm{tot}}}{T_{30}}\right) \approx \frac{K_{\mathrm{eq}} \cdot T_{\mathrm{fold}}}{D_{3 \mathrm{tot}}} .
$$

Integrating this ratio over one dosing cycle at steady state gives

$$
\mathrm{AFTIR}=\frac{1}{\tau} \int_{t_{\mathrm{ss}}}^{t_{\mathrm{ss}}+\tau} \frac{T_{3}(t)}{T_{30}} d t \approx \frac{1}{\tau} \int_{t_{\mathrm{ss}}}^{t_{\mathrm{ss}}+\tau} \frac{K_{\mathrm{eq}} \cdot T_{\mathrm{fold}}}{D_{3 \mathrm{tot}}(t)} d t
$$

When the drug is given as an infusion at a rate Dose $/ \tau$, then the steadystate drug concentration is a constant $\left(D_{3 \text { tot,ss }}\right)$, giving

$$
\mathrm{AFTIR} \approx \frac{K_{\mathrm{eq}} \cdot T_{\mathrm{fold}}}{D_{3 \mathrm{avg}, \mathrm{ss}}}
$$

Substituting the biodistribution expression from Equation 27) gives what we call a simple expression for AFTIR.

$$
\mathrm{AFTIR}_{\mathrm{simple}} \approx \frac{K_{\mathrm{eq}} \cdot T_{\mathrm{fold}}}{B_{\mathrm{sat}}^{\mathrm{ISF}} \cdot C_{\mathrm{avg}}}
$$


$T E C_{50}$ definition

In deriving the above formula for the AFTIR metric, which we call the "simple" AFTIR formula, large drug concentrations are assumed. However, for arbitrarily small drug concentrations, AFTIR simple is unbounded:

$$
\lim _{\text {Dose } \rightarrow 0} \operatorname{AFTIR}_{\text {simple }}=\infty .
$$

Realistically, AFTIR should approach one for arbitrarily small drug concentrations since the average free tissue target at steady state should approach the baseline tissue target concentration. Motivated by [9], we then modify the AFTIR approximation by adding an additional factor to the denominator:

$$
\text { AFTIR } \approx \frac{K_{\mathrm{eq}} \cdot T_{\mathrm{fold}}}{K_{\mathrm{eq}} \cdot T_{\mathrm{fold}}+B_{\mathrm{sat}}^{\mathrm{ISF}} \cdot C_{\mathrm{avg}}}=\frac{T E C_{50}}{T E C_{50}+B_{\mathrm{sat}}^{\mathrm{ISF}} \cdot C_{\mathrm{avg}}},
$$

where the approximate concentration for $50 \%$ target engagement $\left(T E C_{50}\right)$ is given by

$$
T E C_{50}:=K_{\mathrm{eq}} \cdot T_{\mathrm{fold}} \text {. }
$$

$T E C_{50}$ is equivalent to the "L50" parameter in [9]. With this reformulation, we have

$$
\lim _{\text {Dose } \rightarrow 0} \operatorname{AFTIR}=1 .
$$

This new formulation still converges to the previously derived formula such that

$$
\lim _{\text {Dose } \rightarrow \infty} \mathrm{AFTIR}=\mathrm{AFTIR}_{\text {simple }} .
$$

Both the $T E C_{50}$ and simple formulas for AFTIR are checked against numerical simulations in the Results section. We attempted to derive the above expressions for AFTIR by extending the work from [9, but the derivation proved difficult for the model shown here. Thus, we instead posit that this approximation holds and will check it in the following sections.

\section{Definition of TFTIR}

Besides AFTIR, another quantity that can be used to guide dosing regimen is TFTIR (the Trough Free Tissue target to Initial target Ratio). It measures the minimum ratio of the free tissue target (e.g. within a tumor) to the initial tissue target at steady state and is defined as

$$
\text { TFTIR }:=\frac{T_{3, \text { trough }}}{T_{30}}
$$

Like AFTIR, TFTIR can be approximated by

$$
\text { TFTIR } \approx \frac{K_{\mathrm{eq}} \cdot T_{\mathrm{fold}}}{K_{\mathrm{eq}} \cdot T_{\mathrm{fold}}+B_{\mathrm{sat}}^{\mathrm{ISF}} \cdot C_{\mathrm{trough}}}
$$




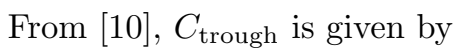

$$
C_{\text {trough }}=\text { Dose } \cdot\left(\frac{C_{1} \cdot \exp (-\alpha \cdot \tau)}{1-\exp (-\alpha \cdot \tau)}+\frac{C_{2} \cdot \exp (-\beta \cdot \tau)}{1-\exp (-\beta \cdot \tau)}+\frac{C_{3} \cdot \exp (-\gamma \cdot \tau)}{1-\exp (-\gamma \cdot \tau)}\right)
$$

where all constants can be computed from the model parameters [11. We will numerically check whether this approximation for TFTIR is accurate in the next section.

\section{Linear PK and $C L$}

In the case that the PK is linear, and there is no elimination from the tumor compartment (i.e., $k_{\mathrm{e} D 3}=0$ ), one can write $C_{\text {avg }}$ in terms of the dosing regimen and the parameters governing the pharmacokinetics: bioavailability $(F)$, clearance $(C L)$, and dosing interval $(\tau)$. It is given by

$$
C_{\text {avg }}=\frac{F \cdot \text { Dose }}{C L \cdot \tau} .
$$

If there is elimination from the tumor (i.e., $k_{\mathrm{e} D 3} \neq 0$ ), then the formula above does not apply with $C L=k_{\mathrm{e} D 1} / V_{D 1}$. However, a similarity transform can be applied where $k_{\mathrm{e} D 3}$ can be forced to be zero, and all the other PK parameters $k_{12 D}, k_{21 D}, k_{13 D}, k_{31 D}, k_{\mathrm{e} D 1}, C L$ can be transformed such that the concentration time profiles for $D_{1}(t)$ and $D_{3}(t)$ do not change. See Appendix A.3 for this similarity transformation. Thus, once $C L$ is estimated by fitting a compartmental model, the formula above for $C_{\text {avg }}$ holds whether or not there is elimination from the tissue compartment.

\section{Methods}

Under the assumption of large doses and a constant infusion of drug, we have arrived at the following expression for AFTIR:

$$
\mathrm{AFTIR} \approx \frac{K_{\mathrm{eq}} \cdot T_{\mathrm{fold}}}{K_{\mathrm{eq}} \cdot T_{\mathrm{fold}}+B \cdot C_{\mathrm{avg}}}
$$

We hypothesize that this expression also holds for TFTIR, replacing $C_{\text {avg }}$ with $C_{\text {trough }}$.

To check the accuracy of this formula, we compare the theoretical AFTIR to the simulated AFTIR. The theoretical AFTIR is computed by Equation (38) for a given set of parameters. The simulated AFTIR is computed directly by simulating Equations (2) - 12) for the same set of parameters and then taking the ratio of the steady state average (or trough) free target concentration to the baseline target concentration. In addition, the Thiele Modulus is also computed using Equation (13). The model is implemented using the RxODE package in $\mathrm{R}$, and correct implementation of the model is checked by comparing the simulated and analytical expressions for $B_{\mathrm{sat}}^{\mathrm{ISF}}, C_{\mathrm{avg}}, C_{\mathrm{trough}}$ and $T_{\mathrm{fold}}$. 
This simulations are performed for four mAbs that are used to treat cancer: trastuzumab (anti-HER2), atezolizumab (anti-PD-L1), pembrolizumab (anti-PD-1), and bevacizumab (anti-VEGF). Key properties of these drugs are summarized in Table (1), and the specific parameters used in this analysis are provided in Table S1. The references and equations used for selecting each parameter are provided in the Excel spreadsheets available here: https://github.com/iamstein/TumorModeling/tree/master/data and in the Supplementary Material. An overview for how the parameters are selected is provided below. For atezolizumab and pembrolizumab, membrane-bound target is shed to form soluble target. For trastuzumab, only membrane-bound target is simulated (shedding is set to zero), while for bevacizumab, only soluble target is simulated (synthesis of membrane-bound target is set to zero).

\section{Parameter Selection}

The PK parameters $\left(k_{\mathrm{e} D 1}, k_{12 D}, k_{21 D}, V_{D 1}, V_{D 2}\right)$ are taken from PopPK model fits from the literature. The volumes for the target tissue are assumed to be $0.1 \mathrm{~L}$, corresponding to a tumor with radius of $3 \mathrm{~cm}$. The binding affinity $\left(K_{\mathrm{d}}\right.$ or $K_{\mathrm{ss}}$ ) is also taken from the literature, and, when needed, a typical value for $k_{\text {off3 }}$ between $1 /$ day-100/day was assumed [12, Figure 12]. The binding and unbinding rates are assumed to be the same in both the central and tissue compartments. For baseline soluble and membrane-bound target levels, data from the literature is used. For membrane-bound targets, target expression $\left(M_{10}\right.$, $\left.M_{30}\right)$ is provided in terms of receptors per cell $(N)$, which is then converted to nanomolar concentration with

$$
M_{i 0}[\mathrm{n} M]=N \cdot \rho_{i} \cdot \phi \cdot \frac{10^{9}}{6.02 \cdot 10^{23}},
$$

where $i=1,3$ refer to the central and target tissue compartments respectively. Here $\rho_{i}$ is the cell density of the tissue of interest $\left(\rho_{1}=6 \cdot 10^{9}\right.$ cells $/ \mathrm{L}$ in blood for targets expressed on white blood cells, and $\rho_{3}=3 \cdot 10^{11}$ cells $/ \mathrm{L}$ for targets expressed in tumor tissue [7), and $\phi$ is the fraction of cells in the tissue of interest expressing the target, assumed to be $0.1-1$.

The target elimination and shedding rates are chosen to have reasonable values (1/day - 10/day) [13], and the synthesis rates are chosen so that baseline target levels matched the estimates for $M_{i 0}$ above.

It is assumed that the rates of distribution of drug into the tumor are proportional to the rates of distribution into the peripheral tissue, scaled by the ratio of tissue volumes:

$$
k_{13 D}=k_{12 D} \cdot \frac{V_{D 3}}{V_{D 2}} .
$$

These estimates were similar to estimates from a more physiological model that accounted for drug perfusion into the tumor 7, as documented in the parameter Excel tables. The biodistribution coefficient $\left(B_{\mathrm{sat}}^{\mathrm{ISF}}\right)$ is assumed to 
be around $30 \%$ 4], and assuming no elimination of drug from the tumor, $k_{31 D}$ is estimated by assuming that at steady state, the transit rates into and out of the tumor are equal:

$$
\begin{aligned}
k_{31 D} \cdot D_{3} \cdot V_{D 3} & =k_{13 D} \cdot D_{1} \cdot V_{D 1}, \\
k_{31 D} \cdot\left(B_{\mathrm{sat}}^{\mathrm{ISF}} \cdot D_{1}\right) \cdot V_{D 3} & =k_{13 D} \cdot D_{1} \cdot V_{D 1}, \\
k_{31 D} & =\frac{k_{13 D}}{B_{\mathrm{sat}}^{\mathrm{ISF}}} \cdot \frac{V_{D 1}}{V_{D 3}} .
\end{aligned}
$$

Soluble targets are then assumed to distribute two times faster than the drug, and soluble drug-taget complexes are assumed to distribute two times slower than the drug. It is recognized that this approach is empirical, and as a check, these rates were found to be comparable to the rates estimated using the Krogh cylinder approach for modeling tissue distribution [14].

For membrane-bound targets, where immune trafficking may occur (pembrolizumab, atezolizumab), it is assumed that the rate of distribution of cells expressing those targets into and out of tissue is $k_{13 M}=0.048 / d$, and $k_{31 M}=$ $5.5 / d$ [15, Table 4 , parameters $k_{\text {in }}$ and $\left.k_{\text {out }}\right]$.

Further parameter exploration

In the above definition of AFTIR, three different approximations are used for the equilibrium binding constant $K_{\text {eq }}$ : quasi-equilibrium $\left(K_{\mathrm{d}}\right)$, quasi-steadystate $\left(K_{\mathrm{ss}}\right)$, and the newly derived constant, quasi-steady-state with distribution $\left(K_{\mathrm{ssd}}\right)$. The accuracy of each of these constants over a range of doses is compared for the four drugs above.

To further check the accuracy of the AFTIR approximation, we varied a few of the 47 model parameters over a large range with the dose fixed at a dose of $10 \mathrm{mg} / \mathrm{kg}$ every three weeks.

$-k_{13 D}$ - the rate of distribution of the drug from the central compartment to the tissue compartment. This will impact the biodistribution $\left(B_{\mathrm{sat}}^{\mathrm{ISF}}\right)$ of drug in the tissue.

$-k_{13 D T}$ - the rate of distribution/trafficking of the drug-target complex from the central compartment to the tissue compartment. This parameter is included because it is often excluded from TMDD models and it was found to impact AFTIR.

- $k_{\mathrm{synT3}}$ - the rate of target synthesis in the tissue compartment. This will impact whether the drug at a particular dose is in excess to its target.

- $k_{\text {shed } D M 3}$ - the rate of shedding of the drug-target complex from membranebound to soluble. This parameter is included because it is also often excluded from TMDD models.

For pembrolizumab, atezolizumab, and trastuzumab, $T$ denotes the membranebound target, and for bevacizumab, $T$ denotes the soluble target.

To determine the importance of an accurate estimate for the soluble target accumulation in the tissue of interest, we also explore how AFTIR varies for 
the parameter $k_{e S 3}$ for bevacizumab, as this parameter has a significant impact on $T_{\text {fold }}=S_{3 \text { tot }, \text { s }} / S_{30}$.

All code for generating the figures shown here is provided at: https:// github.com/iamstein/TumorModeling/tree/master/ModelF.

\section{Results}

AFTIR calculated by simulation is compared to the theoretical formula in Equation (38). The results are shown in Figures 2, 3, and 4).

Figure (2) (top row) shows comparisons of the approximations over a range of doses using the three equilibrium constants, $K_{\mathrm{d}}, K_{\mathrm{ss}}$, and $K_{\mathrm{ssd}}$. In general, $K_{\text {ssd }}$, the new equilibrium constant derived here, best matches the model simulation. Note, however, that for trastuzumab, the drug with the highest target levels, the theory does not match the simulation at all. This is because the AFTIR approximation requires the assumption that the drug is in vast excess to its target and that the tumor can be treated as a homogeneous tissue. The bottom row of Figure (2) shows how the Thiele modulus changes with dose. When the Thiele Modulus is less than 0.2, there is relatively good visual agreement between the theory and the simulation. Because the Thiele modulus is primarily applicable for membrane-bound targets, it is not computed for Bevacizumab.

Note that for Atezolizumab, the agreement improves when the dose drops to $0.1 \mathrm{mg} / \mathrm{kg}$, despite the Thiele Modulus being greater than 0.2 . This is because for any drug given at sufficiently low doses, AFTIR $\approx 1$ for both theory and simulation because no drug is present.

Another feature to note is that the $K_{\mathrm{ss}}$ approximation differs from the $K_{\mathrm{ssd}}$ approximation by less that 2 -fold. Thus, the $K_{\mathrm{ss}}$ approximation is still reasonable, and if one wanted a more conservative estimate for target engagement, a $2 \times$ "safety factor" could multiply $K_{\text {ss }}$. Similar findings are also observed for TFTIR (see supplementary material).

In Figure (3), it is shown that at high doses, AFTIR and AFTIR simple agree well at values below 0.3 . But above 0.3 , and especially at small concentrations where AFTIR approaches 1, the simple approximation no longer holds, as expected.

Figure (4) shows the sensitivity of AFTIR to other parameters, namely the rate of trafficking of the drug from the central compartment to the tissue compartment $\left(k_{13 D}\right)$, the rate of trafficking of the drug-target complex from the central compartment to the tissue compartment $\left(k_{13 D T}\right)$, the rate of shedding of the drug-target complex from membrane-bound to soluble $\left(k_{\text {shed } D M 3}\right)$, and the rate of target synthesis in the tissue compartment $\left(k_{\mathrm{syn} T 3}\right)$. The red horizontal line denotes the regime where the Thiele Modulus is greater than 0.2 , indicating that the assumptions of tissue homogeneity and drug in excess of the target are inaccurate. For bevacizumab and pembrolizumab, there is generally good agreement between theory and simulation. For trastuzumab, the theory does not match the simulation due to the very high target concen- 
tration. And for atezolizumab, the theory matches the simulation only when the Thiele modulus is below 0.2

For atezolizumab, the parameter for which there is the greatest discrepancy between theory and simulation is $k_{\mathrm{syn} T 3}$. At low $k_{\mathrm{syn} T 3}$ values, the theory overpredicts the AFTIR value, while for high $k_{\mathrm{syn} T 3}$ values, the theory underpredicts the AFTIR value. The reason for the discrepancy at high $k_{\mathrm{syn} T 3}$ values is that the target expression becomes so high that the drug is no longer in excess to the target, as in the trastuzumab case. The reason for the discrepancy at low $k_{\mathrm{syn} T 3}$ values is that the assumption of negligible complex transport from circulation into the tissue of interest no longer applies. And so the approximation for deriving $K_{\text {ssd }}$ in Equation (21) is no longer accurate. As a check of this phenomenon, we set $k_{13 D T}$ to zero. As a result, there is better agreement between theory and simulation in Figure (S1).

In Figure (5), it is shown that for a drug (bevacizumab) with a soluble target (VEGF), the fold-accumulation of the target is a critical factor in predicting the amount of target inhibition in the tissue of interest. This is also observed in Equation (31), where AFTIR is directly proportional to $T_{\text {fold }}$.

\section{Discussion}

Review of key AFTIR parameters

The key insight from this work is that under many clinically relevant scenarios, AFTIR can be estimated using four parameters, a binding constant $\left(K_{\mathrm{eq}}\right)$, the fold-change in target levels upon binding to drug ( $\left.T_{\text {fold }}\right)$, the average drug concentration in circulation $\left(C_{\text {avg }}\right)$, and the biodistribution coefficient for the drug to the tissue of interest $\left(B_{\mathrm{sat}}^{\mathrm{ISF}}\right)$ :

$$
\text { AFTIR }=\frac{K_{\mathrm{eq}} \cdot T_{\mathrm{fold}}}{K_{\mathrm{eq}} \cdot T_{\mathrm{fold}}+B_{\mathrm{sat}}^{\mathrm{ISF}} \cdot C_{\mathrm{avg}}} .
$$

This simple formula provides intuition for how changing the dosing regimen, improving the binding affinity of the drug, or enhancing tissue penetration would be expected to alter target inhibition. For example, at large drug concentrations, when AFTIR $\approx \mathrm{AFTIR}_{\text {simple }}=K_{\mathrm{eq}} \cdot T_{\mathrm{fold}} /\left(B_{\mathrm{sat}}^{\mathrm{ISF}} \cdot C_{\mathrm{avg}}\right)$, one can see that either halving the dosing interval, halving the binding affinity (with a more potent drug), or doubling the tissue accessibility (with a drug that enhances tissue penetration) would reduce the free target concentration by approximately 50\%. Even though the model in Figure (1) has many parameters, some of which are difficult to estimate, the AFTIR formula shows that under many practical scenarios, predicting the target inhibition can be done with an estimate of only four lumped parameters. The method for estimating each of these parameters is described below.

The drug concentration $\left(C_{\mathbf{a v g}}\right.$ or $\left.C_{\text {trough }}\right)$ is estimable from PK data from Phase 1 clinical trials. For monoclonal antibodies, it can also be readily predicted from preclinical data [16]. 
The binding affinity $\left(K_{\mathrm{d}}, K_{\mathrm{ss}}\right.$ or $\left.K_{\mathrm{ssd}}\right)$ can either be estimated in vitro with surface plasmon resonance and cell binding assays, or in the clinic with a model-based analysis of soluble target data [1] or receptor occupancy assays [17. Often there is good agreement between the in vitro and in vivo estimates, though it is critical that the assays be carefully validated as there have been instances of 1000-fold differences between the in vitro and in vivo estimates [18, Figure 8]. Also, assays measuring free or bound target concentrations, rather than total target concentrations, can be difficult to validate [19. While $K_{\text {ssd }}$ provided the best match between theory and simulation, it is difficult to measure directly because it requires target occupancy measurements in the tissue of interest. Thus a modeler may also use an estimate for $K_{\mathrm{ss}}$ and, if desired, multiply this binding affinity by a safety factor (e.g. $2 \times$ ) for a more conservative estimate of target engagement.

The biodistribution coefficient ( $B_{\mathbf{s a t}}^{\mathbf{I S F}}$ ) has been estimated for many tissues in monkeys 20, where it ranges from $5-15 \%$ in the total tissue or $15-45 \%$ in the tissue interstitial fluid (assuming that $1 / 3$ of the tissue is interstitial fluid). In mouse tumors, $B_{\mathrm{sat}}^{\mathrm{ISF}}=30 \%$, 4 , and this is similar in human skin for secukinumab [21]. However, others have used predictions of tumor distribution based on rates of extravasation and diffusion to predict that the antibody concentration in a tumor should be only $0.1-1 \%[\underline{6}$. Due to this disagreement in estimates for $B_{\mathrm{sat}}^{\mathrm{ISF}}$ and the limited data in the literature, further work in estimating biodistribution would be of value 22 .

The fold-change in target concentration $\left(T_{\text {fold }}\right)$ in the tissue of interest can be estimated from in vitro experiments for membrane-bound targets by estimating the internalization rates of bound and unbound receptors. It is generally around $T_{\text {fold }}=0.5$ [23, 24, page 15]. Care must be taken when interpreting biopsy staining data from immunohistochemistry (IHC) assays to estimate $T_{\text {fold }}$.

For soluble targets, $T_{\text {fold }}$ is often directly measured in circulation [1, but to our knowledge, it has only been measured once in tissue, namely for IL-6 in mouse synovial fluid 25. This data was difficult to interpret since many measurements were below the limit of quantification of the assay. Therefore, a significant challenge in applying this formula to soluble targets is that the extent of target-accumulation in the tissue of interest is not known. Some of the methods for estimating $B_{\mathrm{sat}}^{\mathrm{ISF}}$ can also be employed for measuring $T_{\text {fold }}$; in particular, techniques such as dermal open flow microperfusion (skin) [21] or microdialysis (tumor) [26] could be employed to measure both drug concentration and soluble target concentration.

Review of assumptions and caveats

The following assumptions are needed for the theoretical estimate of AFTIR to be accurate.

The drug concentration is much larger than the target concentration, and the tissue can be treated as homogeneous. This assumption 
should be checked in two ways. First, by comparing estimates of target expression in the tissue of interest to estimates of the drug concentration in that tissue. And second, for membrane-bound targets, one can check whether the Thiele Modulus is small. 6, 7] For this model, it is found that the Thiele modulus has to be less than 0.2 for the theory to describe the simulation well. The assumption of homogeneity fails for trastuzumab at all doses and for atezolizumab at doses less than $3 \mathrm{mg} / \mathrm{kg} \mathrm{q} 3 \mathrm{w}$. In the future, it would be useful to check whether this threshold of 0.2 holds for other drugs and other models.

Distribution of the drug-target complex from circulation into the tissue is relatively small. This assumption was required for the calculation of $K_{\mathrm{ssd}}$. If distribution of the complex from circulation into the tissue of interest plays a significant role, then the AFTIR formula may not apply. One instance where it may need to be checked is for a soluble target that accumulates 100-1000 times in circulation but not in the tissue of interest. This assumption is difficult to check and requires knowledge of the underlying biology. If it is known that the target is primarily expressed in the tumor, or if its a membranebound target that doesn't move from circulation into the tumor, then this assumption would be reasonable.

The degree of inhibition needed for efficacy is known. Often, 90$95 \%$ inhibition is assumed to be needed [27, though for a drug that works by stimulating the immune system to attack the tumor, much less inhibition may be needed. Examples include trastuzumab, which may also work via antibody dependent cell-mediated cytotoxicity and bispecific T-cell engagers like blinatumomab, which facilitates the interaction between $\mathrm{T}$ cells and cancer cells.

Competition for target binding sites between drug and endogenous ligand. If the binding between a target and its endogenous ligand is much lower than the binding affinity of the drug, the formulas derived here may not applicable because the drug may not be disrupting the interactions between the target and its endogenous ligand. In this analysis, avidity is also not included in the analysis. In the scenario where the monoclonal antibody is in vast excess to the target, each drug molecule is expected to bind at most only one target molecule, and thus avidity was not addressed in this analysis.

Distribution of target and complex to peripheral tissue can be neglected. A more realistic model would include distribution of the drug, target, and complex to each tissue; a lumped, minimal PBPK model could also be considered that also allows for distribution of all components to peripheral tissue. However, we note that this approach using a peripheral compartment for just drug concentrations is often used for predicting tissue distribution [7, 28, 3. Moreover, the expression for $T_{\text {fold }}$ for this model was already quite complex, and thus the analysis of this more complex system is left to future research. 
Applications

Phase 2 dose selection. A key application of this work is to provide an updated methodology for guiding Phase 2 dose selection for biologics when the safety, efficacy, and biomarker data are insufficient to guide dose selection. With this approach, one uses a PopPK model based on Phase 1 PK data to predict the steady state trough concentration of the drug. Then, together with estimates for the binding affinity, fold accumulation of target upon treatment, and biodistribution of drug to tissue, one can apply the AFTIR or TFTIR equation to identify the dose at which, say, $90 \%$ of patients are expected to achieve $90 \%$ target inhibition. While a similar methodology was used previously for atezolizumab [4], $K_{\text {ssd }}$ and $T_{\text {fold }}$ were not explicitly accounted for.

Rapid assessment of drugs. The AFTIR potency metric also allows for a rapid assessment of new drugs without a need for extensive simulations. In particular, given that $\operatorname{AFTIR}_{\text {simple }}=\left(K_{\mathrm{eq}} \cdot T_{\mathrm{fold}} \cdot C L \cdot \tau\right) /\left(F \cdot B_{\mathrm{sat}}^{\mathrm{ISF}} \cdot\right.$ Dose $)$, this formula shows how changing the dosing regimen, clearance, and binding affinity would impact target engagement.

Moreover, rather than specify every rate constant of the complex system of Figure (1), or an even more complex physiological model, it is sufficient in many scenarios to provide estimates for only four parameters: $K_{\mathrm{eq}}, T_{\text {fold }}$, $B_{\mathrm{sat}}^{\mathrm{ISF}}$, and $C_{\mathrm{avg}}$, all of which can in principle be measured directly.

Rapid sensitivity analyses. While all parameters can in principle be measured directly, there will often be scenarios where there is uncertainty about the specific parameter values. In that case, the AFTIR potency metric also allows for a rapid calculation of the impact of this uncertainty on the predicted receptor occupancy. Given the uncertainty, one can then either try to collect additional information about the parameters with the highest degree of uncertainty, or factor this uncertainty into the analysis when informing drug development decisions. For instance, if a drug is relatively safe, one may choose to take forward a higher dose into Phase 2 to ensure that more patients achieve a high degree of target engagement.

\section{Conclusions}

In summary, we have extended previous work to develop potency metrics AFTIR and TFTIR that characterize target inhibition in a tissue, such as a tumor. These metrics predict the target inhibition at steady state under a repeated dosing regimen using four quantities: the average (or trough) drug concentration at steady state, the biodistribution of drug to its target tissue, the equilibrium binding constant, and the fold-accumulation of the target. AFTIR and TFTIR provide intuition for how various physiological properties of the system impact target engagement. In addition, AFTIR and TFTIR can be used for rapid assessment of new targets and exploration of the binding affinity, half-life, bioavailability, and dosing regimen needed for a second-generation drug to achieve comparable or superior efficacy to a marketed compound. Thus 
far, a significant challenge in applying AFTIR is in obtaining accurate, clinical estimates in tissue for biodistribution and fold-accumulation of target in the tissue of interest. It is our hope that this work will motivate scientists to use assays like dermal open flow microperfusion or microdialysis to better estimate these parameters in the future.

\section{Acknowledgements}

The authors would like to thank the IMA as the Math-to-Industry Bootcamp was where this work began. We'd also like to thank Ngartelbaye Guerngar who was also a member of the bootcamp team and Wenping Wang and Matt Fidler for help in using the RxODE package. Finally, we'd like to thank Eduardo Sontag and Greg Thurber for many helpful discussions.

\section{References}

1. A. Stein and R. Ramakrishna, "Afir: A dimensionless potency metric for characterizing the activity of monoclonal antibodies," CPT: pharmacometrics 8 systems pharmacology, vol. 6, no. 4, pp. 258-266, 2017.

2. W. Wang and H. Zhou, "Pharmacological considerations for predicting pk/pd at the site of action for therapeutic proteins," Drug Discovery Today: Technologies, vol. 21, pp. 35-39, 2016.

3. A. Lindauer, C. Valiathan, K. Mehta, V. Sriram, R. de Greef, J. Elassaiss-Schaap, and D. de Alwis, "Translational pharmacokinetic/pharmacodynamic modeling of tumor growth inhibition supports dose-range selection of the anti-pd-1 antibody pembrolizumab," CPT: pharmacometrics \& systems pharmacology, vol. 6, no. 1, pp. 11-20, 2017.

4. R. Deng, D. Bumbaca, C. V. Pastuskovas, C. A. Boswell, D. West, K. J. Cowan, H. Chiu, J. McBride, C. Johnson, Y. Xin, et al., "Preclinical pharmacokinetics, pharmacodynamics, tissue distribution, and tumor penetration of anti-pd-11 monoclonal antibody, an immune checkpoint inhibitor," MAbs, vol. 8, no. 3, pp. 593-603, 2016.

5. D. E. Mager and W. J. Jusko, "General pharmacokinetic model for drugs exhibiting target-mediated drug disposition," Journal of pharmacokinetics and pharmacodynamics, vol. 28, no. 6, pp. 507-532, 2001.

6. G. M. Thurber, M. M. Schmidt, and K. D. Wittrup, "Antibody tumor penetration: transport opposed by systemic and antigen-mediated clearance," Advanced drug delivery reviews, vol. 60 , no. 12 , pp. 1421-1434, 2008.

7. G. M. Thurber and K. D. Wittrup, "A mechanistic compartmental model for total antibody uptake in tumors," Journal of theoretical biology, vol. 314, pp. 57-68, 2012.

8. P. Ma, "Theoretical considerations of target-mediated drug disposition models: simplifications and approximations," Pharmaceutical research, vol. 29, no. 3, pp. 866-882, 2012.

9. J. Gabrielsson and L. A. Peletier, "Pharmacokinetic steady-states highlight interesting target-mediated disposition properties," The AAPS journal, vol. 19, no. 3, pp. 772-786, 2017.

10. M. Rowland and T. Tozer, "Clinical pharmacokinetics/pharmacodynamics," 2005.

11. F. M. Anne Dubois, Julie Bertrand, "Mathematical expressions of the pharmacokinetic and pharmacodynamic models implemented in the pfim software." 2011.

12. D. Yang, A. Singh, H. Wu, and R. Kroe-Barrett, "Comparison of biosensor platforms in the evaluation of high affinity antibody-antigen binding kinetics," Analytical biochemistry, vol. 508, pp. 78-96, 2016. 
13. N. Li, C. J. Workman, S. M. Martin, and D. A. Vignali, "Biochemical analysis of the regulatory t cell protein lymphocyte activation gene-3 (lag-3; cd223)," The Journal of Immunology, vol. 173, no. 11, pp. 6806-6812, 2004.

14. C. Cilliers, H. Guo, J. Liao, N. Christodolu, and G. M. Thurber, "Multiscale modeling of antibody-drug conjugates: connecting tissue and cellular distribution to whole animal pharmacokinetics and potential implications for efficacy," The AAPS journal, vol. 18, no. 5, pp. 1117-1130, 2016.

15. V. S. Ayyar, S. Sukumaran, D. C. DuBois, R. R. Almon, J. Qu, and W. J. Jusko, "Receptor/gene/protein-mediated signaling connects methylprednisolone exposure to metabolic and immune-related pharmacodynamic actions in liver," Journal of Pharmacokinetics and Pharmacodynamics, vol. 45, pp. 557-575, Aug 2018.

16. R. Deng, S. Iyer, F.-P. Theil, D. L. Mortensen, P. J. Fielder, and S. Prabhu, "Projecting human pharmacokinetics of therapeutic antibodies from nonclinical data: what have we learned?," in MAbs, vol. 3, pp. 61-66, Taylor \& Francis, 2011.

17. M. Liang, M. Schwickart, A. K. Schneider, I. Vainshtein, C. Del Nagro, N. Standifer, and L. K. Roskos, "Receptor occupancy assessment by flow cytometry as a pharmacodynamic biomarker in biopharmaceutical development," Cytometry Part B: Clinical Cytometry, vol. 90, no. 2, pp. 117-127, 2016.

18. G. M. Meno-Tetang and P. J. Lowe, "On the prediction of the human response: a recycled mechanistic pharmacokinetic/pharmacodynamic approach," Basic ES clinical pharmacology $\&$ toxicology, vol. 96, no. 3, pp. 182-192, 2005.

19. J. W. Lee, M. Kelley, L. E. King, J. Yang, H. Salimi-Moosavi, M. T. Tang, J.-F. Lu, J. Kamerud, A. Ahene, H. Myler, et al., "Bioanalytical approaches to quantify "total" and "free" therapeutic antibodies and their targets: technical challenges and pk/pd applications over the course of drug development," The AAPS journal, vol. 13, no. 1, pp. 99-110, 2011

20. D. K. Shah and A. M. Betts, "Antibody biodistribution coefficients: inferring tissue concentrations of monoclonal antibodies based on the plasma concentrations in several preclinical species and human," MAbs, vol. 5, no. 2, pp. 297-305, 2013.

21. C. Dragatin, F. Polus, M. Bodenlenz, C. Calonder, B. Aigner, K. I. Tiffner, J. K. Mader, M. Ratzer, R. Woessner, T. R. Pieber, et al., "Secukinumab distributes into dermal interstitial fluid of psoriasis patients as demonstrated by open flow microperfusion," Experimental dermatology, vol. 25, no. 2, pp. 157-159, 2016

22. I. H. Bartelink, E. F. Jones, S. K. Shahidi-Latham, E. L. Rong, Y. Zheng, P. Vicini, L. v. Veer, D. Wolf, A. Iagaru, D. L. Kroetz, B. Prideaux, C. Cilliers, G. Thurber, Z. Wimana, and G. Geraldine, "Tumor drug penetration measurements could be the neglected piece of the personalized cancer treatment puzzle," Clinical Pharmacology and Therapeutics, 2018.

23. B.-F. Krippendorff, K. Kuester, C. Kloft, and W. Huisinga, "Nonlinear pharmacokinetics of therapeutic proteins resulting from receptor mediated endocytosis," Journal of pharmacokinetics and pharmacodynamics, vol. 36, no. 3, pp. 239-260, 2009.

24. V. Knutson, "Ligand-independent internalization and recycling of the insulin receptor effects of chronic treatment of 3t3-c2 fibroblasts with insulin and dexamethasone.," Journal of Biological Chemistry, vol. 267, no. 2, pp. 931-937, 1992.

25. X. Chen, X. Jiang, W. J. Jusko, H. Zhou, and W. Wang, "Minimal physiologically-based pharmacokinetic (mpbpk) model for a monoclonal antibody against interleukin-6 in mice with collagen-induced arthritis," Journal of pharmacokinetics and pharmacodynamics, vol. 43 , no. 3 , pp. $291-304,2016$.

26. Q. Zhou and J. M. Gallo, "In vivo microdialysis for pk and pd studies of anticancer drugs," The AAPS Journal, vol. 7, pp. E659-E667, Sep 2005.

27. S. Grimwood and P. R. Hartig," "Target site occupancy: emerging generalizations from clinical and preclinical studies," Pharmacology 83 therapeutics, vol. 122, no. 3, pp. 281$301,2009$.

28. A. Tiwari, A. K. Abraham, J. M. Harrold, A. Zutshi, and P. Singh, "Optimal affinity of a monoclonal antibody: Guiding principles using mechanistic modeling," The AAPS Journal, pp. 1-10, 2016.

29. K. R. Godfrey and M. J. Chapman, "The problem of model indistinguishability in pharmacokinetics," Journal of Pharmacokinetics and Biopharmaceutics, vol. 17, pp. 229267, Apr 1989. 


\section{Appendix}

Fold-Accumulation $\left(T_{\text {fold }}\right)$

Recall that the fold-accumulation of target is given by

$$
T_{\text {fold }}:=\frac{T_{3 \text { tot }, \mathrm{ss}}}{T_{30}}=\left\{\begin{array}{ll}
\frac{M_{3 \mathrm{tot}, \mathrm{ss}}}{M_{30}}, & \text { for membrane-bound targets } \\
\frac{S_{3 \mathrm{tot}, \mathrm{ss}}}{S_{30}}, & \text { for soluble targets }
\end{array},\right.
$$

where $T_{3 \text { tot,ss }}$ denotes the sum of target and drug-target complex concentration at steady state, and $T_{30}$ denotes target concentration at the initial state. In the sections below, we solve for the baseline target and the steady state target (for large drug concentrations) using similar approaches. Then, we compute $T_{\text {fold }}$ as the ratio of the two.

\section{Baseline target ( $T_{10}$ and $\left.T_{30}\right)$}

Before the drug is given, the system is at steady state. And we have $D_{1}=D_{2}=D_{3}=$ $D M_{1}=D M_{3}=D S_{1}=D S_{3}=0$. Using the ODE system, we solve for the nonzero initial membrane-bound targets. Setting Equations $(5)$ and $(6)$ to zero, we get a system of two linear equations,

$$
\begin{aligned}
& \left(\begin{array}{l}
0 \\
0
\end{array}\right)=\frac{d}{d t}\left(\begin{array}{l}
M_{1} \\
M_{3}
\end{array}\right) \\
& =\left(\begin{array}{cc}
-\left(k_{\mathrm{shed} M_{1}}+k_{13 M}+k_{\mathrm{e} M 1}\right) & \left(V_{D 3} / V_{D 1}\right) k_{31 M} \\
\left(V_{D 1} / V_{D 3}\right) k_{13 M} & -\left(k_{\mathrm{shed} M_{3}}+k_{31 M}+k_{\mathrm{e} M 3}\right)
\end{array}\right)\left(\begin{array}{l}
M_{1} \\
M_{3}
\end{array}\right)_{0}+\left(\begin{array}{l}
k_{\mathrm{synM} 1} \\
k_{\mathrm{synM} 3}
\end{array}\right) .
\end{aligned}
$$

Rearranging yields

$$
\left(\begin{array}{cc}
\left(k_{\mathrm{shed} M_{1}}+k_{13 M}+k_{\mathrm{e} M 1}\right) & -\left(V_{D 3} / V_{D 1}\right) k_{31 M} \\
-\left(V_{D 1} / V_{D 3}\right) k_{13 M} & \left(k_{\mathrm{shed} M_{3}}+k_{31 M}+k_{\mathrm{e} M 3}\right)
\end{array}\right)\left(\begin{array}{c}
M_{1} \\
M_{3}
\end{array}\right)_{0}=\left(\begin{array}{c}
k_{\mathrm{synM} 1} \\
k_{\mathrm{synM} 3}
\end{array}\right)
$$

Then, using the formula for inverting a $2 \mathrm{D}$ matrix gives

$$
\begin{aligned}
& M_{10}=\frac{\left(k_{\mathrm{shed} M_{3}}+k_{31 M}+k_{\mathrm{e} M 3}\right) k_{\mathrm{synM} 1}+\left(V_{D 3} / V_{D 1}\right) k_{31 M} k_{\mathrm{synM} 3}}{\left(k_{\mathrm{shed} M_{1}}+k_{13 M}+k_{\mathrm{e} M 1}\right)\left(k_{\mathrm{shed} M_{3}}+k_{31 M}+k_{\mathrm{e} M 3}\right)-k_{31 M} k_{13 M}}, \\
& M_{30}=\frac{\left(V_{D 1} / V_{D 3}\right) k_{13 M} k_{\mathrm{synM} 1}+\left(k_{\mathrm{shed} M_{1}}+k_{13 M}+k_{\mathrm{e} M 1}\right) k_{\mathrm{synM} 3}}{\left(k_{\mathrm{shed} M_{1}}+k_{13 M}+k_{\mathrm{e} M 1}\right)\left(k_{\mathrm{shed} M_{3}}+k_{31 M}+k_{\mathrm{e} M 3}\right)-k_{31 M} k_{13 M}} .
\end{aligned}
$$

To solve for the nonzero initial soluble targets, we set Equations (7) and 8) to zero, resulting in a system of two linear equations,

$$
\begin{aligned}
& \left(\begin{array}{l}
0 \\
0
\end{array}\right)=\frac{d}{d t}\left(\begin{array}{l}
S_{1} \\
S_{3}
\end{array}\right) \\
& =\left(\begin{array}{cc}
-\left(k_{13 S}+k_{\mathrm{e} S 1}\right) & \left(V_{D 3} / V_{D 1}\right) k_{31 S} \\
\left(V_{D 1} / V_{D 3}\right) k_{13 S} & -\left(k_{31 S}+k_{\mathrm{e} S 3}\right)
\end{array}\right)\left(\begin{array}{c}
S_{1} \\
S_{3}
\end{array}\right)_{0}+\left(\begin{array}{c}
k_{\mathrm{synS}_{1}}+k_{\mathrm{shedM}_{1}} \\
k_{\mathrm{synS} 3}+k_{\mathrm{shedM}_{3}}
\end{array}\right) .
\end{aligned}
$$

Similar to above, we get

$$
\begin{aligned}
& S_{10}=\frac{\left(k_{31 S}+k_{\mathrm{e} S 3}\right)\left(k_{\mathrm{synS} 1}+k_{\mathrm{shedM}_{1}} M_{10}\right)+\left(V_{D 3} / V_{D 1}\right) k_{31 S}\left(k_{\mathrm{synS} 3}+k_{\mathrm{shedM}_{3}} M_{30}\right)}{\left(k_{13 S}+k_{\mathrm{e} S 1}\right)\left(k_{31 S}+k_{\mathrm{e} S 3}\right)-k_{31 S} k_{13 S}}, \\
& S_{30}=\frac{\left(V_{D 1} / V_{D 3}\right) k_{13 S}\left(k_{\mathrm{synS} 1}+k_{\mathrm{shedM}_{1}} M_{10}\right)+\left(k_{13 S}+k_{\mathrm{e} S 1}\right)\left(k_{\mathrm{synS} 3}+k_{\mathrm{shedM}_{3}} M_{30}\right)}{\left(k_{13 S}+k_{\mathrm{e} S 1}\right)\left(k_{31 S}+k_{\mathrm{e} S 3}\right)-k_{31 S} k_{13 S}},
\end{aligned}
$$

where $M_{10}$ and $M_{30}$ are given by 41 and 42 . 
Steady State Value

After the drug is given, the system reaches steady state. We assume that the drug is in vast excess to the amount of target so that almost all of the target is bound to the drug. Then we have $M_{1}=M_{3}=S_{1}=S_{3} \approx 0, M_{3 \mathrm{tot}, \mathrm{ss}}=M_{3}+D M_{3}$, and $S_{3 \text { tot,ss }}=S_{3}+D S_{3}$. Using the ODE system, we solve for the nonzero steady state of the total membrane-bound targets. Adding the equations for the target and complex, Equations (5) and 6) added to Equations 9 and 100 , respectively, gives differential equations for the total target that are similar to that when no drug is on board. We set these differential equations to zero, resulting in a system of two linear equations. We solve as in the previous section to get

$M_{1 \mathrm{tot}, \mathrm{ss}}=\frac{\left(k_{\mathrm{shed} D M_{3}}+k_{31 D M}+k_{\mathrm{e} D M 3}\right) k_{\mathrm{synM} 1}+\left(V_{D 3} / V_{D 1}\right) k_{31 D M} k_{\mathrm{synM} 3}}{\left(k_{\mathrm{shed} D M_{1}}+k_{13 D M}+k_{\mathrm{e} D M 1}\right)\left(k_{\mathrm{shed} D M_{3}}+k_{31 D M}+k_{\mathrm{e} D M 3}\right)-k_{31 D M} k_{13 D M}}$,

$M_{3 \mathrm{tot}, \mathrm{ss}}=\frac{\left(V_{D 1} / V_{D 3}\right) k_{13 D M} k_{\mathrm{synM} 1}+\left(k_{\mathrm{shed} D M_{1}}+k_{13 D M}+k_{\mathrm{e} D M 1}\right) k_{\mathrm{synM} 3}}{\left(k_{\mathrm{shed} D M_{1}}+k_{13 D M}+k_{\mathrm{e} D M 1}\right)\left(k_{\mathrm{shed} D M_{3}}+k_{31 D M}+k_{\mathrm{e} D M 3}\right)-k_{31 D M} k_{13 D M}}$

Likewise, the nonzero steady state of total soluble targets are given by

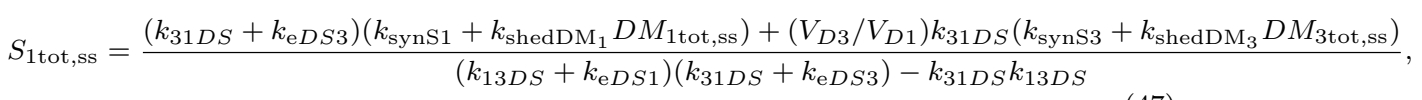

$S_{3 \mathrm{tot}, \mathrm{ss}}=\frac{\left(V_{D 1} / V_{D 3}\right) k_{13 D S}\left(k_{\mathrm{synS} 1}+k_{\mathrm{shedDM}_{1}} D M_{1 \mathrm{tot}, \mathrm{ss}}\right)+\left(k_{13 D S}+k_{\mathrm{e} D S 1}\right)\left(k_{\mathrm{synS} 3}+k_{\mathrm{shedDM}_{3}} D M_{3 \mathrm{tot}, \mathrm{ss}}\right)}{\left(k_{13 D S}+k_{\mathrm{e} D S 1}\right)\left(k_{31 D S}+k_{\mathrm{e} D S 3}\right)-k_{31 D S} k_{13 D S}}$,

where $M_{1 \text { tot,ss }}$ and $M_{3 \text { tot,ss }}$ are given by 45 and 46 .

A summary of all the of equations is provided below. To calculate $T_{\text {fold }}$, one then uses the formulas below to compute $M_{3 \text { tot,ss }} / M_{30}$ or $S_{3 \text { tot,ss }} / S_{30}$. 


$$
\begin{aligned}
M_{10} & =\frac{\left(k_{\mathrm{shed} M_{3}}+k_{31 M}+k_{\mathrm{e} M 3}\right) k_{\mathrm{synM} 1}+\left(V_{D 3} / V_{D 1}\right) k_{31 M} k_{\mathrm{synM} 3}}{\left(k_{\mathrm{shed} M_{1}}+k_{13 M}+k_{\mathrm{e} M 1}\right)\left(k_{\mathrm{shed} M_{3}}+k_{31 M}+k_{\mathrm{e} M 3}\right)-k_{31 M} k_{13 M}} \\
M_{30} & =\frac{\left(V_{D 1} / V_{D 3}\right) k_{13 M} k_{\mathrm{synM} 1}+\left(k_{\mathrm{shed} M_{1}}+k_{13 M}+k_{\mathrm{e} M 1}\right) k_{\mathrm{synM} 3}}{\left(k_{\mathrm{shed} M_{1}}+k_{13 M}+k_{\mathrm{e} M 1}\right)\left(k_{\mathrm{shed} M_{3}}+k_{31 M}+k_{\mathrm{e} M 3}\right)-k_{31 M} k_{13 M}} \\
S_{10} & =\frac{\left(k_{31 S}+k_{\mathrm{e} S 3}\right)\left(k_{\mathrm{synS} 1}+k_{\mathrm{shedM}} M_{10}\right)+\left(V_{D 3} / V_{D 1}\right) k_{31 S}\left(k_{\mathrm{synS} 3}+k_{\mathrm{shedM}_{3}} M_{30}\right)}{\left(k_{13 S}+k_{\mathrm{e} S 1}\right)\left(k_{31 S}+k_{\mathrm{e} S 3}\right)-k_{31 S} k_{13 S}} \\
S_{30} & =\frac{\left(V_{D 1} / V_{D 3}\right) k_{13 S}\left(k_{\mathrm{synS} 1}+k_{\mathrm{shedM}} M_{10}\right)+\left(k_{13 S}+k_{\mathrm{e} S 1}\right)\left(k_{\mathrm{synS} 3}+k_{\mathrm{shedM}_{3}} M_{30}\right)}{\left(k_{13 S}+k_{\mathrm{e} S 1}\right)\left(k_{31 S}+k_{\mathrm{e} S 3}\right)-k_{31 S} k_{13 S}}
\end{aligned}
$$

$$
M_{1 \mathrm{tot}, \mathrm{ss}}=\frac{\left(k_{\mathrm{shed} D M_{3}}+k_{31 D M}+k_{\mathrm{e} D M 3}\right) k_{\mathrm{synM} 1}+\left(V_{D 3} / V_{D 1}\right) k_{31 D M} k_{\mathrm{synM} 3}}{\left(k_{\mathrm{shed} D M_{1}}+k_{13 D M}+k_{\mathrm{e} D M 1}\right)\left(k_{\mathrm{shed} D M_{3}}+k_{31 D M}+k_{\mathrm{e} D M 3}\right)-k_{31 D M} k_{13 D M}}
$$

$$
M_{3 \mathrm{tot}, \mathrm{ss}}=\frac{\left(V_{D 1} / V_{D 3}\right) k_{13 D M} k_{\mathrm{synM} 1}+\left(k_{\mathrm{shed} D M_{1}}+k_{13 D M}+k_{\mathrm{e} D M 1}\right) k_{\mathrm{synM} 3}}{\left(k_{\mathrm{shed} D M_{1}}+k_{13 D M}+k_{\mathrm{e} D M 1}\right)\left(k_{\mathrm{shed} D M_{3}}+k_{31 D M}+k_{\mathrm{e} D M 3}\right)-k_{31 D M} k_{13 D M}}
$$

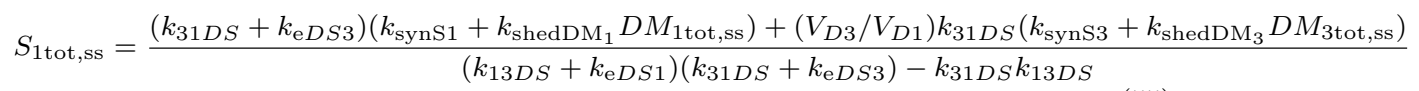

$S_{3 \mathrm{tot}, \mathrm{ss}}=\frac{\left(V_{D 1} / V_{D 3}\right) k_{13 D S}\left(k_{\mathrm{synS} 1}+k_{\mathrm{shedDM}_{1}} D M_{1 \mathrm{tot}, \mathrm{ss}}\right)+\left(k_{13 D S}+k_{\mathrm{e} D S 1}\right)\left(k_{\mathrm{synS} 3}+k_{\mathrm{shedDM}_{3}} D M_{3 \mathrm{tot}, \mathrm{ss}}\right)}{\left(k_{13 D S}+k_{\mathrm{e} D S 1}\right)\left(k_{31 D S}+k_{\mathrm{e} D S 3}\right)-k_{31 D S} k_{13 D S}}$

Biodistribution $\left(B_{\text {sat }}^{\mathrm{ISF}}\right)$

For very large infusion where the target concentration is negligable, we can write the three equations for the drug $\left(D_{1}, D_{2}, D_{3}\right)$ from Equations [2], (3), and (4) as below to solve for steady state.

$$
\begin{aligned}
-\left(\begin{array}{c}
k_{\mathrm{inf}} \\
0 \\
0
\end{array}\right) & =\frac{d}{d t}\left(\begin{array}{c}
D_{1} \\
D_{2} \\
D_{3}
\end{array}\right) \\
& =\left(\begin{array}{ccc}
-\left(k_{\mathrm{e} D 1}+k_{12 D}+k_{13 D}\right) & \left(V_{D 2} / V_{D 1}\right) k_{21 D} & \left(V_{D 3} / V_{D 1}\right) k_{31 D} \\
\left(V_{D 1} / V_{D 2}\right) k_{12 D} & -k_{21 D} & 0 \\
\left(V_{D 1} / V_{D 3}\right) k_{13 D} & 0 & -\left(k_{\mathrm{e} D 3}+k_{31 D}\right)
\end{array}\right) \cdot\left(\begin{array}{c}
D_{1} \\
D_{2} \\
D_{3}
\end{array}\right) \\
& =A \cdot\left(\begin{array}{c}
D_{1} \\
D_{2} \\
D_{3}
\end{array}\right) .
\end{aligned}
$$

$B_{\mathrm{sat}}^{\mathrm{ISF}}$ is given by the ratio of $D_{3, s s} / D_{1, s s}$ where $s s$ denotes steady state, such that when the drug concentration is large

$$
D_{3 \text { tot }, \text { avg }}=B_{\mathrm{sat}}^{\mathrm{ISF}} \cdot D_{1 \mathrm{tot}, \mathrm{avg}}=B_{\mathrm{sat}}^{\mathrm{ISF}} \cdot C_{\mathrm{avg}},
$$

where we define $C_{\text {avg }}:=D_{1 \text { tot,avg. }}$. 
$B_{\text {sat }}^{\mathrm{ISF}}$ is computed as follows:

$$
\begin{aligned}
\left(\begin{array}{c}
D_{1} \\
D_{2} \\
D_{3}
\end{array}\right)_{s s} & =A^{-1} \cdot\left(\begin{array}{c}
-k_{\mathrm{inf}} \\
0 \\
0
\end{array}\right) \\
& =\frac{-k_{\inf }}{\operatorname{det} A}\left(\begin{array}{c}
a_{22} a_{33}-a_{23} a_{32} \\
a_{23} a_{31}-a_{21} a_{33} \\
a_{21} a_{32}-a_{22} a_{31}
\end{array}\right)=\frac{-k_{\inf }}{\operatorname{det} A}\left(\begin{array}{c}
k_{21 D}\left(k_{\mathrm{e} D 3}+k_{31 D}\right) \\
k_{21 D}\left(V_{D 2} / V_{D 1}\right)\left(k_{e D 3}+k_{31 D}\right) \\
k_{21 D}\left(V_{D 1} / V_{D 3}\right) k_{13 D}
\end{array}\right) .
\end{aligned}
$$

Thus the biodistribution coefficient for the tissue interstitial fluid compartment is given by

$$
B_{\mathrm{sat}}^{\mathrm{ISF}}:=\left(\frac{D_{3}}{D_{1}}\right)_{s s}=\frac{k_{21 D}\left(V_{D 1} / V_{D 3}\right) k_{13 D}}{k_{21 D}\left(k_{\mathrm{e} D 3}+k_{31 D}\right)}=\frac{V_{D 1}}{V_{D 3}} \cdot \frac{k_{13 D}}{\left(k_{\mathrm{e} D 3}+k_{31 D}\right)}
$$

For drugs dosed with linear PK at regular intervals, drug concentration much larger than target concentration, and free drug elimination occuring only in the central compartment, we have $D_{1 \text { tot.avg }}=$ Dose $/(C L \cdot \tau)$, where $C L=k_{\mathrm{e} D 1} \cdot V_{D 1}$ is the drug clearance. As shown in Section A.3 we can derive a similar formula with coefficient matrix $A^{\prime}$ in which $k_{\mathrm{e} D 3}=0$, while $D_{1}^{\prime}$ and $D_{3}^{\prime}$ in the new model are the same as $D_{1}$ and $D_{3}$ in the original model. From Section A.3 we have

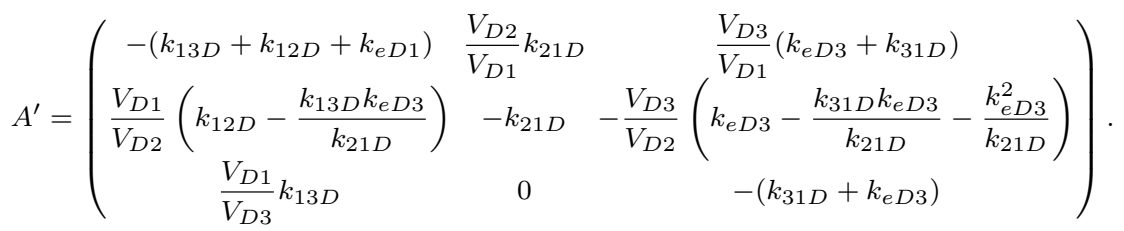

Then, as above,

$$
\begin{aligned}
& \left(\begin{array}{c}
D_{1}^{\prime} \\
D_{2}^{\prime} \\
D_{3}^{\prime}
\end{array}\right)_{s s}=\frac{-k_{\inf }}{\operatorname{det} A^{\prime}}\left(\begin{array}{c}
a_{22} a_{33}-a_{23} a_{32} \\
a_{23} a_{31}-a_{21} a_{33} \\
a_{21} a_{32}-a_{22} a_{31}
\end{array}\right) \\
& =\frac{-k_{\inf }}{\operatorname{det} A^{\prime}}\left(\begin{array}{c}
k_{21 D}\left(k_{31 D}+k_{\mathrm{e} D 3}\right) \\
\left(V_{D 1} / V_{D 2}\right)\left(k_{12 D} k_{31 D}+\frac{k_{12 D}}{k_{e D 3}}+\frac{k_{13 D}}{k_{e D 3}}\right) \\
\left(V_{D 1} / V_{D 3}\right) k_{21 D} k_{13 D}
\end{array}\right)
\end{aligned}
$$

And the biodistribution coefficient for the tissue interstitial fluid compartment is given by

$$
B_{\mathrm{sat}}^{\mathrm{ISF}^{\prime}}:=\left(\frac{D_{3}^{\prime}}{D_{1}^{\prime}}\right)_{s s}=\frac{\left(V_{D 1} / V_{D 3}\right) k_{21 D} k_{13 D}}{k_{21 D}\left(k_{31 D}+k_{\mathrm{e} D 3}\right)}=\frac{V_{D 1}}{V_{D 3}} \cdot \frac{k_{13 D}}{\left(k_{31 D}+k_{\mathrm{e} D 3}\right)},
$$

which is the same as $B_{\mathrm{sat}}^{\mathrm{ISF}}$ in the original model above.

\section{Similarity Transform}

We consider the model in Figure (1). A drug $(D)$ binds to its target $(M)$ to form a complex $(D M)$. It has three compartments, central, tissue, and peripheral. 
The drug and target dynamics are modeled with the following system of ordinary differential equations

$$
\begin{aligned}
\frac{d D_{1}}{d t} & =\frac{1}{V_{C}} \operatorname{Dose}_{i v}(t)-k_{12 D} D_{1}+\frac{V_{P}}{V_{C}} k_{21 D} D_{2}-k_{13 D} D_{1} \\
& +\frac{V_{T}}{V_{C}} k_{31 D} D_{3}-k_{\mathrm{on} 1} D_{1} \cdot M_{1}+k_{\mathrm{off} 1}\left(D M_{1}\right)-k_{\mathrm{e} D 1} D_{1} \\
\frac{d D_{2}}{d t} & =k_{12 D} \frac{V_{C}}{V_{P}} D_{1}-k_{21 D} D_{2} \\
\frac{d D_{3}}{d t} & =\frac{V_{C}}{V_{T}} k_{13 D} D_{1}-k_{31 D} D_{3}-k_{\mathrm{on} 3} D_{3} \cdot M_{3}+k_{\mathrm{off} 3}\left(D M_{3}\right)-k_{\mathrm{e} D 3} D_{3} \\
\frac{d M_{1}}{d t} & =k_{\mathrm{syn} 1}-k_{13 M} M_{1}+\frac{V_{T}}{V_{C}} k_{31 M} M_{3}-k_{\mathrm{on} 1} D_{1} \cdot M_{1}+k_{\mathrm{off} 1}\left(D M_{1}\right) \\
& -k_{\mathrm{e} M 1} M_{1} \\
\frac{d M_{3}}{d t} & =k_{\mathrm{syn} 3}+\frac{V_{C}}{V_{T}} k_{13 M} M_{1}-k_{31 M} M_{3}-k_{\mathrm{on} 3} D_{3} \cdot M_{3}+k_{\mathrm{off} 3}\left(D M_{3}\right) \\
& -k_{\mathrm{e} M 3} M_{3} \\
\frac{d\left(D M_{1}\right)}{d t} & =-k_{13 D M}\left(D M_{1}\right)+\frac{V_{T}}{V_{C}} k_{31 D M}\left(D M_{3}\right)+k_{\mathrm{on} 1} D_{1} \cdot M_{1}-k_{\mathrm{off} 1}\left(D M_{1}\right) \\
& -k_{\mathrm{e} D M 1}\left(D M_{1}\right) \\
\frac{d\left(D M_{3}\right)}{d t} & =\frac{V_{C}}{V_{T}} k_{13 D M}\left(D M_{1}\right)-k_{31 D M}\left(D M_{3}\right)+k_{\mathrm{on} 3} D_{3} \cdot M_{3}-k_{\mathrm{off} 3}\left(D M_{3}\right) \\
& -k_{\mathrm{e} D M 3}\left(D M_{3}\right)
\end{aligned}
$$

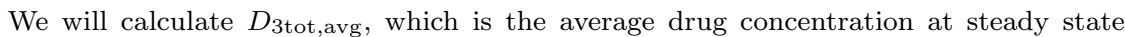

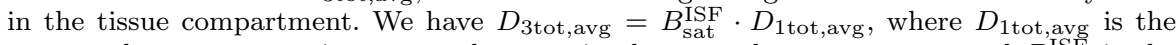
average drug concentration at steady state in the central compartment, and $B_{\mathrm{sat}}^{\mathrm{ISF}}$ is the antibody biodistribution coefficient. Assume that drug elimination only occurs in the central compartment, i.e., $k_{\mathrm{e} D 3}=0$. Then for drugs dosed with linear PK at regular intervals and concentration much larger than target concentration, we have $D_{1 \text { tot,avg }}=\operatorname{Dose} /(C L \cdot \tau)$, where $C L=k_{\mathrm{e} D 1} \cdot V_{C}$ is the drug clearance [10].

For the model with drug elimination in both the central and tissue compartments, we will derive an alternative model with $k_{\mathrm{e} D 3}=0$ that is indistinguishable from the existing model by using the similarity transform technique 29]. We consider drug concentration large enough that target binding does not affect drug distribution. Then the model equations for the drug can be written as

$$
\frac{d \mathbf{D}}{d t}=A \cdot \mathbf{D}(t)+B_{\mathrm{sat}}^{\mathrm{ISF}} \cdot \operatorname{Dose}_{i v}(t)
$$

with measurement

$$
\mathbf{m}(t)=C \cdot \mathbf{D}(t)
$$

where

$$
\begin{gathered}
\mathbf{D}=\left(\begin{array}{c}
D_{1} \\
D_{2} \\
D_{3}
\end{array}\right), \\
A=\left(\begin{array}{ccc}
-\left(k_{13 D}+k_{12 D}+k_{\mathrm{e} D 1}\right) & \frac{V_{P}}{V_{C}} k_{21 D} & \frac{V_{T}}{V_{C}} k_{31 D} \\
\frac{V_{C}}{V_{P}} k_{12 D} & -k_{21 D} & 0 \\
\frac{V_{C}}{V_{T}} k_{13 D} & 0 & -\left(k_{31 D}+k_{\mathrm{e} D 3}\right)
\end{array}\right),
\end{gathered}
$$




$$
\begin{gathered}
B_{\mathrm{sat}}^{\mathrm{ISF}}=\left(\begin{array}{c}
\frac{1}{V_{C}} \\
0 \\
0
\end{array}\right), \\
C=\left(\begin{array}{lll}
1 & 0 & 0 \\
0 & 0 & 1
\end{array}\right) .
\end{gathered}
$$

We transform this model into an indistinguishable model with coefficient matrices $A^{\prime}$, $B_{\mathrm{sat}}^{\mathrm{ISF}^{\prime}}$, and $C^{\prime}$ such that $k_{\mathrm{e} D 3}^{\prime}=0$. This is accomplished with the similarity transform

$$
A^{\prime}:=T A T^{-1}, \quad B_{\mathrm{sat}^{\mathrm{ISF}}}^{\mathrm{I}}:=T B, \quad C^{\prime}:=C T^{-1},
$$

for some transformation matrix

$$
T=\left(\begin{array}{lll}
t_{11} & t_{12} & t_{13} \\
t_{21} & t_{22} & t_{23} \\
t_{31} & t_{32} & t_{33}
\end{array}\right), \quad T^{-1}=\left(\begin{array}{ccc}
\hat{t}_{11} & \hat{t}_{12} & \hat{t}_{13} \\
\hat{t}_{21} & \hat{t}_{22} & \hat{t}_{23} \\
\hat{t}_{31} & \hat{t}_{32} & \hat{t}_{33}
\end{array}\right)
$$

Alternatively, one can get this similarity transform with the change of variables $\mathbf{D}=T^{-1} \mathbf{D}^{\prime}$. Then from 65 and 66, we have

$$
\frac{d\left(T^{-1} \mathbf{D}^{\prime}\right)}{d t}=A \cdot\left(T^{-1} \mathbf{D}^{\prime}\right)(t)+B_{\mathrm{sat}}^{\mathrm{ISF}} \cdot \operatorname{Dose}_{i v}(t), \quad \mathbf{m}(t)=C \cdot\left(T^{-1} \mathbf{D}^{\prime}\right)(t) .
$$

Multiplying the first equation in 73 on the left by $T$ yields

$$
\frac{d \mathbf{D}^{\prime}}{d t}=T A T^{-1} \cdot \mathbf{D}^{\prime}(t)+T B \cdot \operatorname{Dose}_{i v}(t) .
$$

Then we have the new model equation and measurement

$$
\frac{d \mathbf{D}^{\prime}}{d t}=A^{\prime} \cdot \mathbf{D}^{\prime}(t)+B_{\mathrm{sat}}^{\mathrm{ISF}} \cdot \operatorname{Dose}_{i v}(t), \quad \mathbf{m}(t)=C^{\prime} \cdot \mathbf{D}^{\prime}(t),
$$

where $A^{\prime}, B_{\mathrm{sat}}^{\mathrm{ISF}}$, and $C^{\prime}$ are given by 71 .

Regardless of model formulation, the same dose is given to $D_{1}$, that is,

$$
B_{\mathrm{sat}}^{\mathrm{ISF}}=B_{\mathrm{sat}}^{\mathrm{ISF}} .
$$

From this and 717, we get $t_{11}=1, t_{21}=0$, and $t_{31}=0$. Regardless of model formulation, we measure $D_{1}$ and $D_{3}$, that is,

$$
C^{\prime}=C
$$

From this and 71 , we get $\hat{t}_{11}=1, \hat{t}_{12}=0, \hat{t}_{13}=0, \hat{t}_{31}=0, \hat{t}_{32}=0$, and $\hat{t}_{33}=1$. For a $3 \times 3$ matrix, the inverse is given by

$$
T^{-1}=\frac{1}{\operatorname{det} T}\left(\begin{array}{lll}
t_{22} t_{33}-t_{23} t_{32} & t_{13} t_{32}-t_{12} t_{33} & t_{12} t_{23}-t_{13} t_{22} \\
t_{23} t_{31}-t_{21} t_{33} & t_{11} t_{33}-t_{13} t_{31} & t_{13} t_{21}-t_{11} t_{23} \\
t_{21} t_{32}-t_{22} t_{31} & t_{12} t_{31}-t_{11} t_{32} & t_{11} t_{22}-t_{12} t_{21}
\end{array}\right),
$$

with the determinant given by

$$
\operatorname{det} T=t_{11}\left(t_{22} t_{33}-t_{23} t_{32}\right)-t_{12}\left(t_{21} t_{33}-t_{23} t_{31}\right)+t_{13}\left(t_{21} t_{32}-t_{22} t_{31}\right) .
$$

Putting the above findings into $T^{-1}$ yields $t_{12}=0, t_{13}=0, t_{32}=0$, and $t_{33}=1$. From $T T^{-1}=I$, we get $t_{22}=1$. Then we have

$$
T=\left(\begin{array}{ccc}
1 & 0 & 0 \\
0 & 1 & t_{23} \\
0 & 0 & 1
\end{array}\right) \quad T^{-1}=\left(\begin{array}{ccc}
1 & 0 & 0 \\
0 & 1 & -t_{23} \\
0 & 0 & 1
\end{array}\right) .
$$


Putting this into 71 yields

$$
A^{\prime}=\left(\begin{array}{ccc}
-\left(k_{13 D}+k_{12 D}+k_{\mathrm{e} D 1}\right) & \frac{V_{P}}{V_{C}} k_{21 D} & -\frac{V_{P}}{V_{C}} k_{21 D} t_{23}+\frac{V_{T}}{V_{C}} k_{31 D} \\
\frac{V_{C}}{V_{P}} k_{12 D}+\frac{V_{C}}{V_{T}} k_{13 D} t_{23} & -k_{21 D} & \left(k_{21 D}-k_{31 D}-k_{\mathrm{e} D 3}\right) t_{23} \\
\frac{V_{C}}{V_{T}} k_{13 D} & 0 & -\left(k_{31 D}+k_{\mathrm{e} D 3}\right)
\end{array}\right) .
$$

Now we impose

$$
k_{\mathrm{e} D 3}^{\prime}:=-a_{33}^{\prime}-\frac{V_{C}}{V_{T}} a_{13}^{\prime}=0
$$

from which we get $t_{23}=-\left(V_{T} / V_{P}\right)\left(k_{\mathrm{e} D 3} / k_{21 D}\right)$. After putting this last piece into $A^{\prime}$, we have

$$
A^{\prime}=\left(\begin{array}{ccc}
-\left(k_{13 D}+k_{12 D}+k_{\mathrm{e} D 1}\right) & \frac{V_{P}}{V_{C}} k_{21 D} & \frac{V_{T}}{V_{C}}\left(k_{\mathrm{e} D 3}+k_{31 D}\right) \\
\frac{V_{C}}{V_{P}}\left(k_{12 D}-\frac{k_{13 D} k_{\mathrm{e} D 3}}{k_{21 D}}\right) & -k_{21 D} & -\frac{V_{T}}{V_{P}}\left(\begin{array}{c}
\left.k_{\mathrm{e} D 3}-\frac{k_{31 D} k_{\mathrm{e} D 3}}{k_{21 D}}-\frac{k_{\mathrm{e} D 3}^{2}}{k_{21 D}}\right) \\
\frac{V_{C}}{V_{T}} k_{13 D}
\end{array}\right)^{-\left(k_{31 D}+k_{\mathrm{e} D 3}\right)}
\end{array}\right) .
$$

We get $k_{\mathrm{e} D 1}^{\prime}$ from

$$
\begin{aligned}
k_{\mathrm{e} D 1}^{\prime} & =-a_{11}^{\prime}-\frac{V_{P}}{V_{C}} a_{21}^{\prime}-\frac{V_{T}}{V_{C}} a_{31}^{\prime} \\
& =k_{\mathrm{e} D 1}-\frac{k_{13 D} \cdot k_{\mathrm{e} D 3}}{k_{21 D}} .
\end{aligned}
$$

Thus $C L^{\prime}=k_{\mathrm{e} D 1}^{\prime} \cdot V_{C}$, with $k_{\mathrm{e} D 1}^{\prime}$ given by 84$)$. And finally we get $D_{\text {tot,avg1 }}=$ Dose $/\left(C L^{\prime}\right.$. $\tau)$, and $D_{\text {tot,avg3 }}=B_{\mathrm{sat}}^{\mathrm{ISF}} \cdot D_{\mathrm{tot}, \text { avg1 }}$.

Remark: In the similarity transform, we measure $D_{1}$ and $D_{3}$, which is indicated by $C$. As a result of imposing $C^{\prime}=C$, the original model and the transformed model output the same values for these variables. $D_{2}^{\prime}$, however, differs from $D_{2}$.

\section{Figures and Tables}

\begin{tabular}{|l|c|c|c|c|}
\hline Drug & Bevacizumab & Pembrolizumab & Atezolizumab & Trastuzumab \\
\hline Target Type & Soluble & Membrane-Bound & Membrane-Bound & Membrane-Bound \\
\hline Target & VEGF & PD-1 & PD-L1 & HER-2 \\
\hline Baseline Target $(\mathrm{nM})$ & 0.01 & 0.2 & 3 & 800 \\
\hline Shedding Included & no & yes & yes & no \\
\hline
\end{tabular}

Table 1 Summary of drugs in this analysis. Details on all model parameters are provided in the Supplementary Material. 


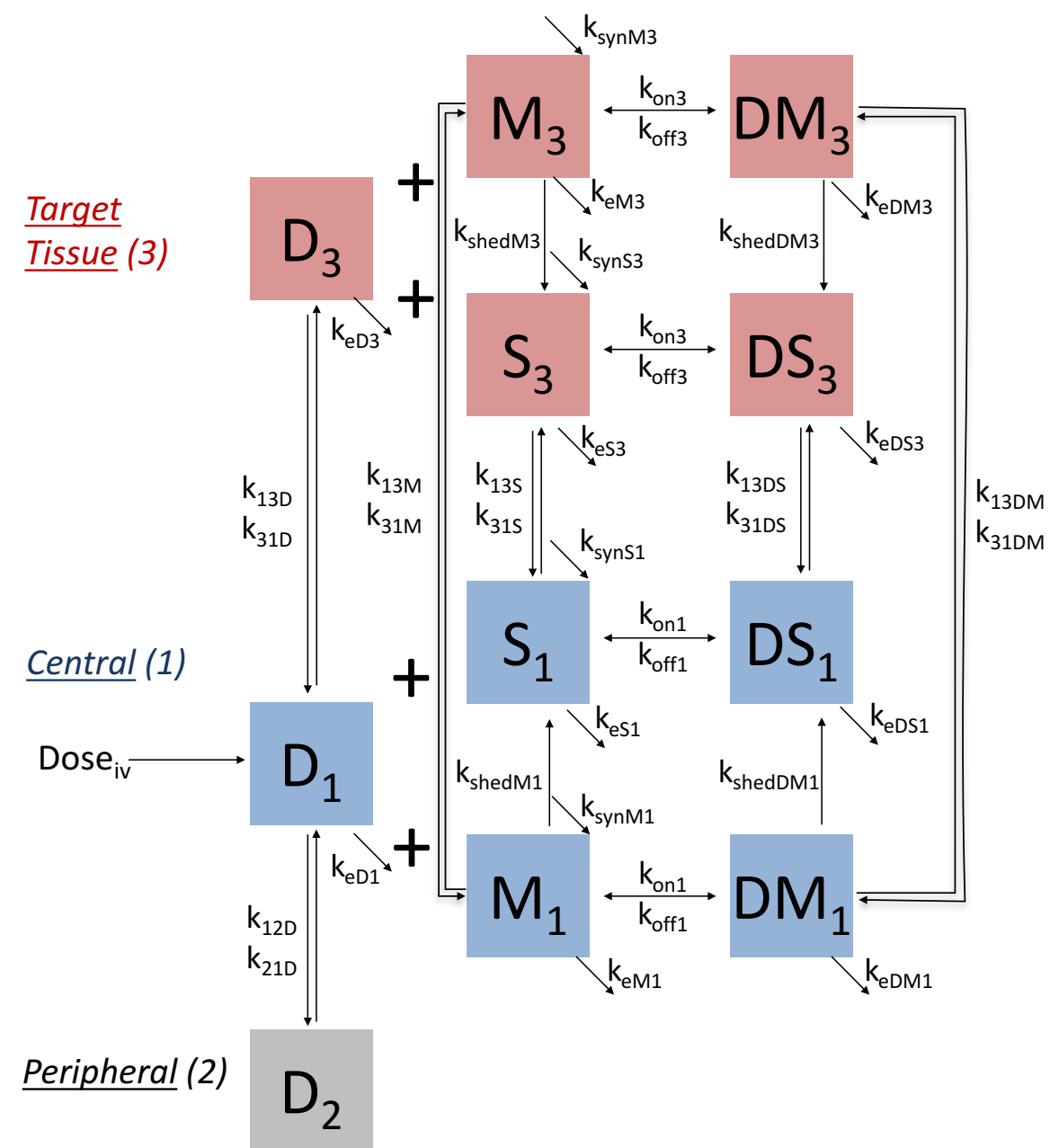

Fig. 1 The extended target mediated drug disposition model. Vertical arrows represent distribution, horizontal arrows represent dosing and binding, and diagonal arrows represent synthesis and elimination. 
bioRxiv preprint doi: https://doi.org/10.1101/432500; this version posted February 6, 2019. The copyright holder for this preprint (which was not certified by peer review) is the author/funder, who has granted bioRxiv a license to display the preprint in perpetuity. It is made available under aCC-BY-NC 4.0 International license.

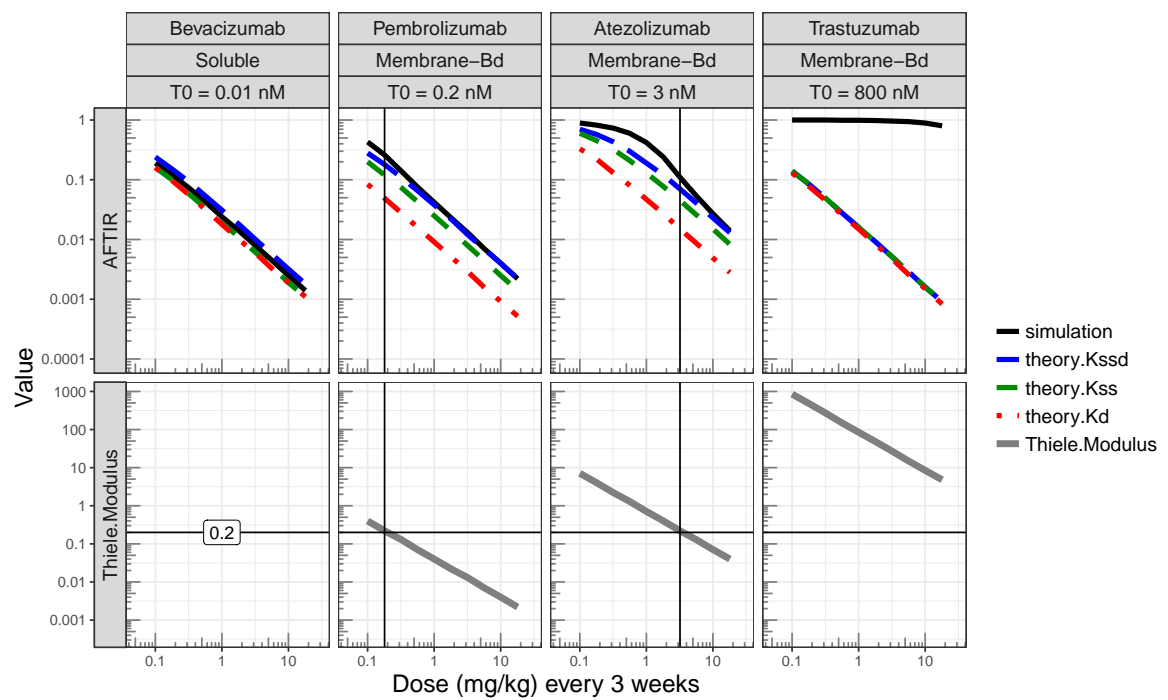

Fig. 2 Average Free Tissue target to Initial target Ratio (AFTIR, top row) and Thiele Modulus ( $\phi^{2}$, bottom row) for four drugs with different baseline target expression levels $\left(T_{0}\right)$ over a range of doses. The $K_{\mathrm{ssd}}$ approximation matches the simulation better than $K_{\mathrm{ss}}$ and $K_{\mathrm{d}}$. When the Thiele Modulus is less than 0.2, the AFTIR $K_{\mathrm{ssd}}$ approximation matches the data well. For trastuzumab in particular, $\phi^{2}>10$, target expression is high $(800 \mathrm{nM})$, and so the theory does not match the simulation. 
bioRxiv preprint doi: https://doi.org/10.1101/432500; this version posted February 6, 2019. The copyright holder for this preprint (which was not certified by peer review) is the author/funder, who has granted bioRxiv a license to display the preprint in perpetuity. It is made available under aCC-BY-NC 4.0 International license.

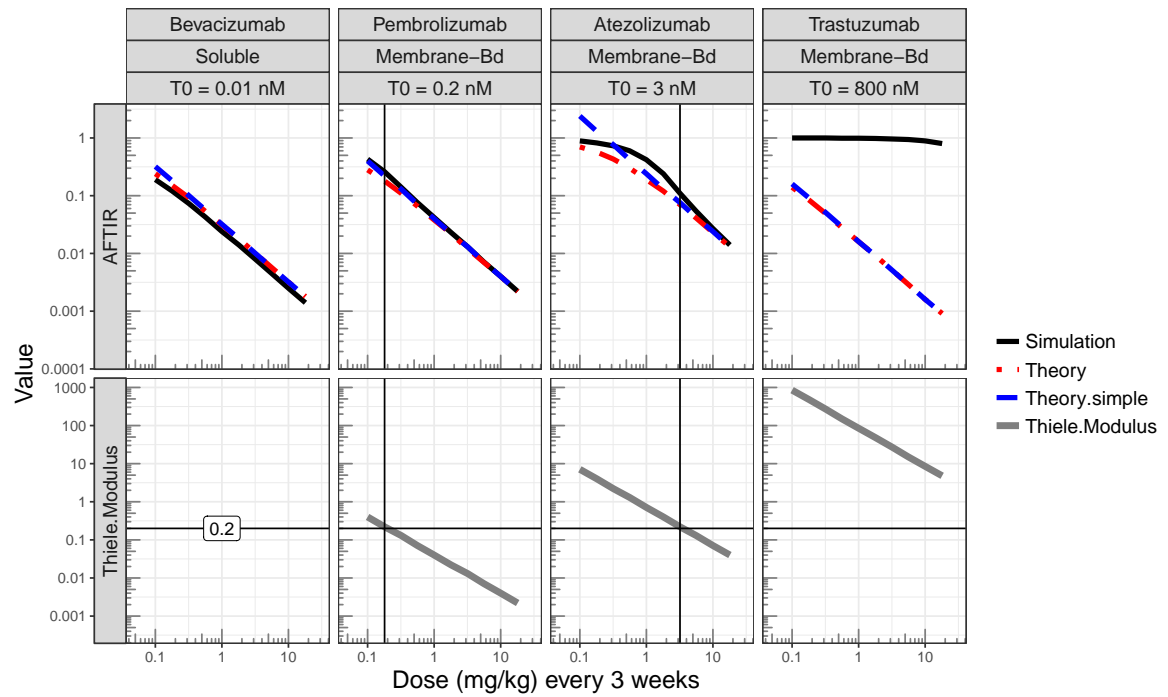

Fig. 3 Average Free Tissue target to Initial target Ratio (AFTIR, top row) and Thiele Modulus ( $\phi^{2}$, bottom row) for four drugs with different baseline target expression levels $\left(T_{0}\right)$ over a range of doses. The AFTIR approximation better matches the simulation than

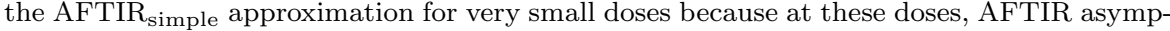
totically approaches 1 , whereas AFTIR simple is unbounded. 
bioRxiv preprint doi: https://doi org/10.1101/432500; this version posted February 6, 2019. The copyright holder for this preprint (which was not certified by peer review) is the author/funder, who has granted bioRxiv a license to display the preprint in perpetuity. It is made available under aCC-BY-NC 4.0 International license.

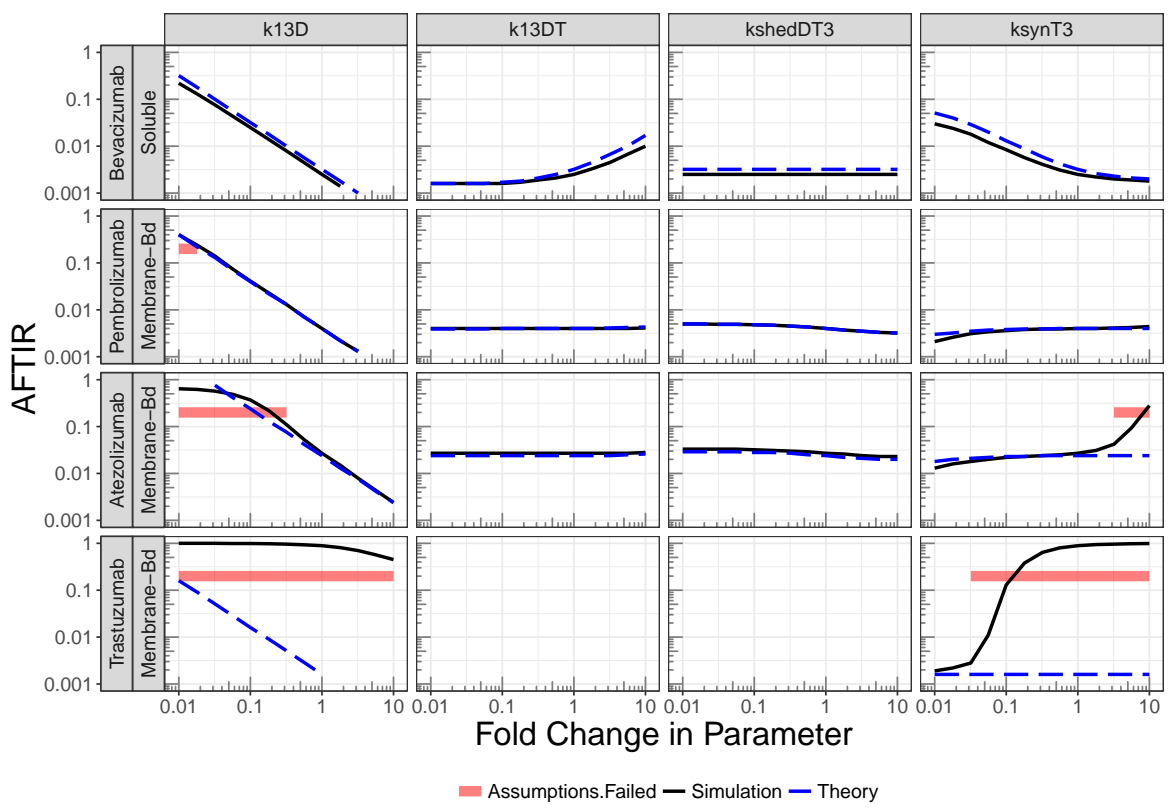

Fig. 4 Exploration of how other parameters impact the Average Free Tissue target to Initial target Ratio (AFTIR) using the $K_{\mathrm{ssd}}$ approximation. The parameter at the top of each column is changed from $0.01 \times$ to $10 \times$ from its baseline value. The red line indicates the regime where the assumption of tissue homogeneity does not hold. Here, this assumption is checked by testing whether the Thiele Modulus is greater than 0.2 . It is in the regime covered by the red line that the theory and simulation do not agree. 
bioRxiv preprint doi: https://doi.org/10.1101/432500; this version posted February 6, 2019. The copyright holder for this preprint (which was not certified by peer review) is the author/funder, who has granted bioRxiv a license to display the preprint in perpetuity. It is made available under aCC-BY-NC 4.0 International license.

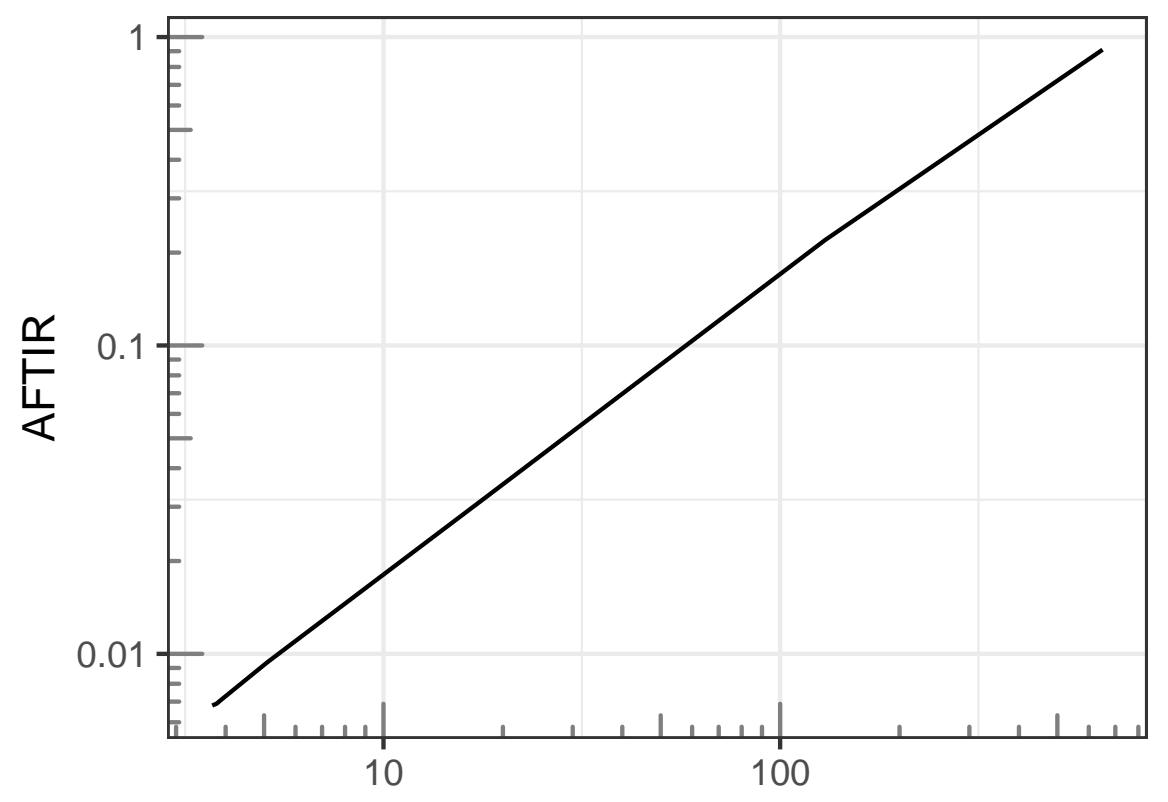

Target Accumulation (Tfold)

Fig. 5 Simulated Average Free Tissue target to Initial target Ratio (AFTIR) as a function of fold change in target $\left(T_{\text {fold }}\right)$ for Bevacizumab.

\section{Supplementary Material}

All parameters and code for executing the model are stored here: https://github.com/ iamstein/TumorModeling 
Model Parameters

\begin{tabular}{|c|c|c|c|c|c|}
\hline Parameter & Units & Atezolizumab & Bevacizumab & Pembrolizumab & Trastuzumab \\
\hline$k_{12 D}$ & $1 / \mathrm{d}$ & 0.17 & 0.43 & 0.23 & 0.17 \\
\hline$k_{13 D}$ & $1 / \mathrm{d}$ & 0.0046 & 0.014 & 0.0056 & 0.013 \\
\hline$k_{13 D M}$ & $1 / \mathrm{d}$ & 0.048 & 0 & 0.048 & 0 \\
\hline$k_{13 D S}$ & $1 / \mathrm{d}$ & 0.0046 & 0.014 & 0.0056 & 0 \\
\hline$k_{13 M}$ & $1 / \mathrm{d}$ & 0.048 & 0 & 0.048 & 0 \\
\hline$k_{13 S}$ & $1 / \mathrm{d}$ & 0.0092 & 0.028 & 0.011 & 0 \\
\hline$k_{21 D}$ & $1 / \mathrm{d}$ & 0.15 & 0.45 & 0.2 & 0.28 \\
\hline$k_{31 D}$ & $1 / \mathrm{d}$ & 0.45 & 1.3 & 0.59 & 0.83 \\
\hline$k_{31 D M}$ & $1 / \mathrm{d}$ & 5.5 & 0 & 5.5 & 0 \\
\hline$k_{31 D S}$ & $1 / \mathrm{d}$ & 0.45 & 1.3 & 0.59 & 1 \\
\hline$k_{31 M}$ & $1 / \mathrm{d}$ & 5.5 & 0 & 5.5 & 0 \\
\hline$k_{31 S}$ & $1 / \mathrm{d}$ & 0.9 & 2.7 & 1.2 & 1 \\
\hline$k_{\mathrm{e} D 1}$ & $1 / \mathrm{d}$ & 0.061 & 0.056 & 0.063 & 0.1 \\
\hline$k_{\mathrm{e} D 3}$ & $1 / \mathrm{d}$ & 0 & 0 & 0 & 0 \\
\hline$k_{\mathrm{e} D M 1}$ & $1 / \mathrm{d}$ & 0 & 1 & 0 & 1 \\
\hline$k_{\mathrm{e} D M 3}$ & $1 / \mathrm{d}$ & 0 & 1 & 0 & 2.9 \\
\hline$k_{\mathrm{e} D S 1}$ & $1 / \mathrm{d}$ & 0.005 & 0.07 & 0.063 & 1 \\
\hline$k_{\mathrm{e} D S 3}$ & $1 / \mathrm{d}$ & 0 & 0.07 & 0 & 1 \\
\hline$k_{\mathrm{e} M 1}$ & $1 / \mathrm{d}$ & 0 & 1 & 0 & 1 \\
\hline$k_{\mathrm{e} M 3}$ & $1 / \mathrm{d}$ & 0 & 1 & 0 & 2.9 \\
\hline$k_{\mathrm{e} S 1}$ & $1 / \mathrm{d}$ & 0.3 & 7 & 0.47 & 1 \\
\hline$k_{\mathrm{e} S 3}$ & $1 / \mathrm{d}$ & 0 & 7 & 0 & 1 \\
\hline$k_{\text {off } 1}$ & $1 / d$ & 3 & 2 & 3.5 & 30 \\
\hline$k_{\mathrm{off} 3}$ & $1 / \mathrm{d}$ & 3 & 2 & 3.5 & 30 \\
\hline$k_{\mathrm{on} 1}$ & $1 /\left(\mathrm{nM}^{*} \mathrm{~d}\right)$ & 1.2 & 36 & 8.2 & 60 \\
\hline$k_{\mathrm{on} 3}$ & $1 /\left(\mathrm{nM}^{*} \mathrm{~d}\right)$ & 1.2 & 36 & 8.2 & 60 \\
\hline$k_{\text {shedDM1 }}$ & $1 / \mathrm{d}$ & 6 & 1 & 6 & 0 \\
\hline$k_{\text {shedDM3 }}$ & $1 / \mathrm{d}$ & 6 & 1 & 6 & 0 \\
\hline$k_{\text {shedM1 }}$ & $1 / \mathrm{d}$ & 3 & 1 & 3 & 0 \\
\hline$k_{\text {shedM } 3}$ & $1 / \mathrm{d}$ & 3 & 1 & 3 & 0 \\
\hline$k_{\text {synM1 }}$ & $\mathrm{nM} / \mathrm{d}$ & 0.45 & 0 & 0.03 & 0 \\
\hline$k_{\mathrm{synM} 3}$ & $\mathrm{nM} / \mathrm{d}$ & 22 & 0 & 1.5 & 2400 \\
\hline$k_{\mathrm{synS} 1}$ & $\mathrm{nM} / \mathrm{d}$ & 0 & 0.014 & 0 & 0 \\
\hline$k_{\text {synS3 }}$ & $\mathrm{nM} / \mathrm{d}$ & 0 & 0.1 & 0 & 0 \\
\hline$V_{D 1}$ & $\mathrm{~L}$ & 3.3 & 3.2 & 3.5 & 2.1 \\
\hline$V_{D 2}$ & $\mathrm{~L}$ & 3.6 & 3.1 & 4.1 & 1.3 \\
\hline$V_{D 3}$ & $\mathrm{~L}$ & 0.1 & 0.1 & 0.1 & 0.1 \\
\hline$V_{D M 1}$ & L & 3.3 & 3.2 & 3.5 & 2.1 \\
\hline$V_{D M 3}$ & L & 0.1 & 0.1 & 0.1 & 0.1 \\
\hline$V_{D S 1}$ & L & 3.3 & 3.2 & 3.5 & 2.1 \\
\hline$V_{D S 3}$ & $\mathrm{~L}$ & 0.1 & 0.1 & 0.1 & 0.1 \\
\hline$V_{M 1}$ & L & 3.3 & 3.2 & 3.5 & 2.1 \\
\hline$V_{M 3}$ & L & 0.1 & 0.1 & 0.1 & 0.1 \\
\hline$V_{S 1}$ & $\mathrm{~L}$ & 3.3 & 3.2 & 3.5 & 2.1 \\
\hline$V_{S 3}$ & L & 0.1 & 0.1 & 0.1 & 0.1 \\
\hline
\end{tabular}

Table S1 Parameters used for the simulations. To find the references and calculations for how these parameters were derived, consult https://github.com/iamstein/TumorModeling/ tree/master/data 
bioRxiv preprint doi: https://doi.org/10.1101/432500; this version posted February 6, 2019. The copyright holder for this preprint (which was not certified by peer review) is the author/funder, who has granted bioRxiv a license to display the preprint in perpetuity. It is made available under aCC-BY-NC 4.0 International license.

AFTIR Simulations

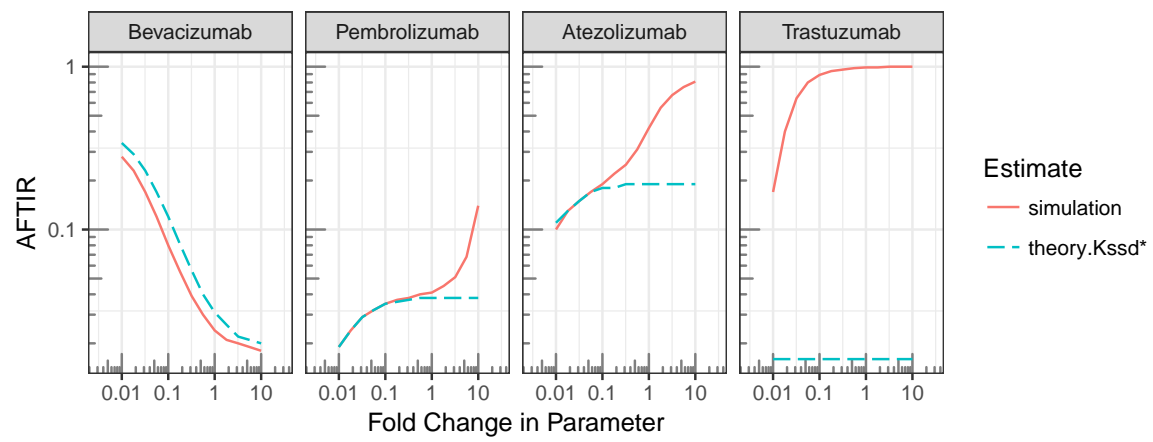

Fig. S1 Parameter exploration for $k_{\mathrm{syn} T 3} . k_{13 D T}$ has been set to zero gives better agreement for $k_{\mathrm{syn} T 3}$. Here, the parameter at the top of each column is changed from $0.01 \times$ to $10 \times$ from its baseline value. There is in general good agreement between theory and experiment, except for trastuzumab, when the target expression is high.

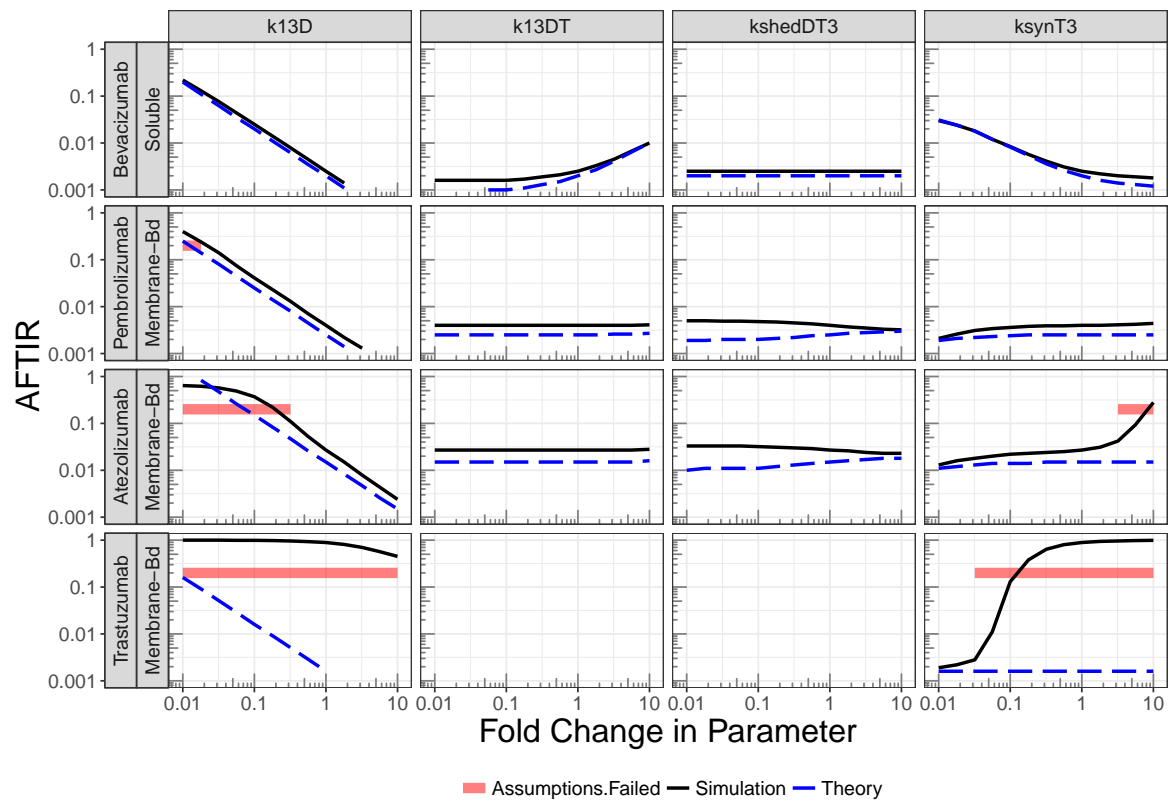

Fig. S2 Parameter exploration where the parameter at the top of each column is changed from $0.01 \times$ to $10 \times$ from its baseline value. There is in general good agreement between theory and experiment, except for trastuzumab, when the target expression is high. Kss approximation. 
bioRxiv preprint doi: https://doi.org/10.1101/432500; this version posted February 6, 2019. The copyright holder for this preprint (which was not certified by peer review) is the author/funder, who has granted bioRxiv a license to display the preprint in perpetuity. It is made available under aCC-BY-NC 4.0 International license.

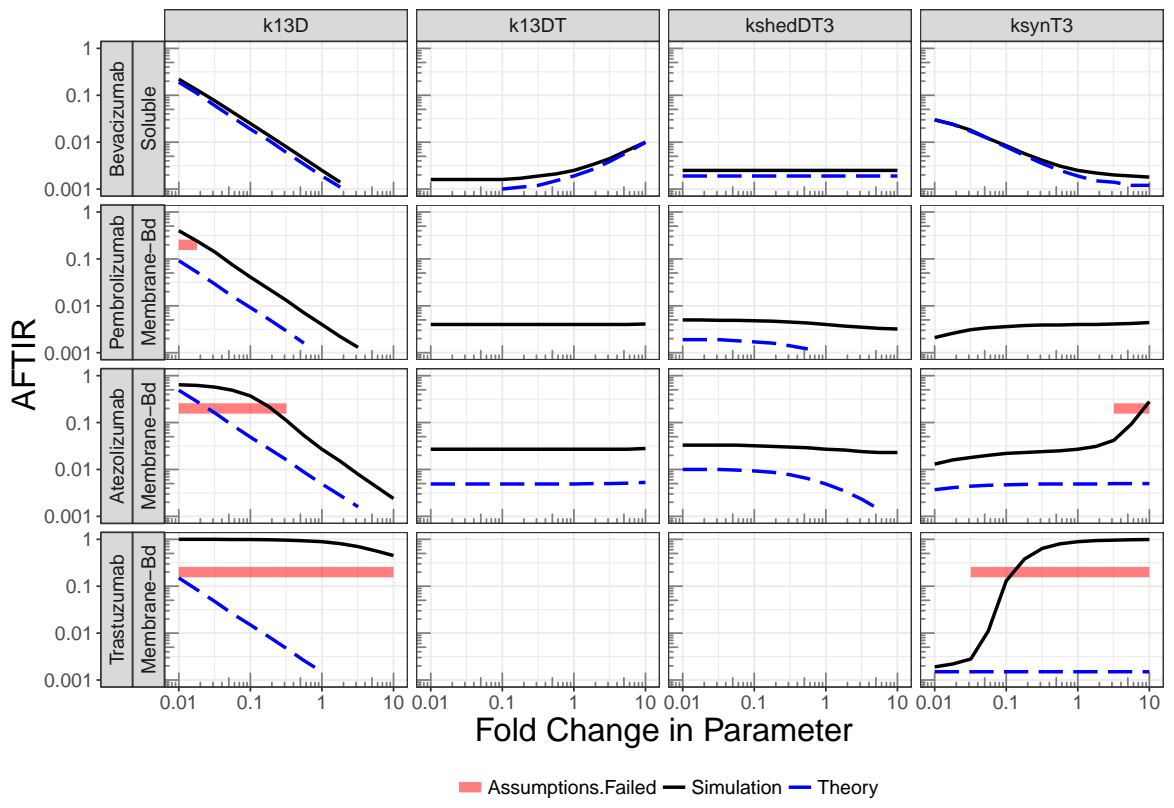

Fig. S3 Parameter exploration where the parameter at the top of each column is changed from $0.01 \times$ to $10 \times$ from its baseline value. There is in general good agreement between theory and experiment, except for trastuzumab, when the target expression is high. Kd approximation. 
bioRxiv preprint doi: https://doi.org/10 1101/432500; this version posted February 6, 2019. The copyright holder for this preprint (which was not certified by peer review) is the author/funder, who has granted bioRxiv a license to display the preprint in perpetuity. It is made available under aCC-BY-NC 4.0 International license.

TFTIR simulations

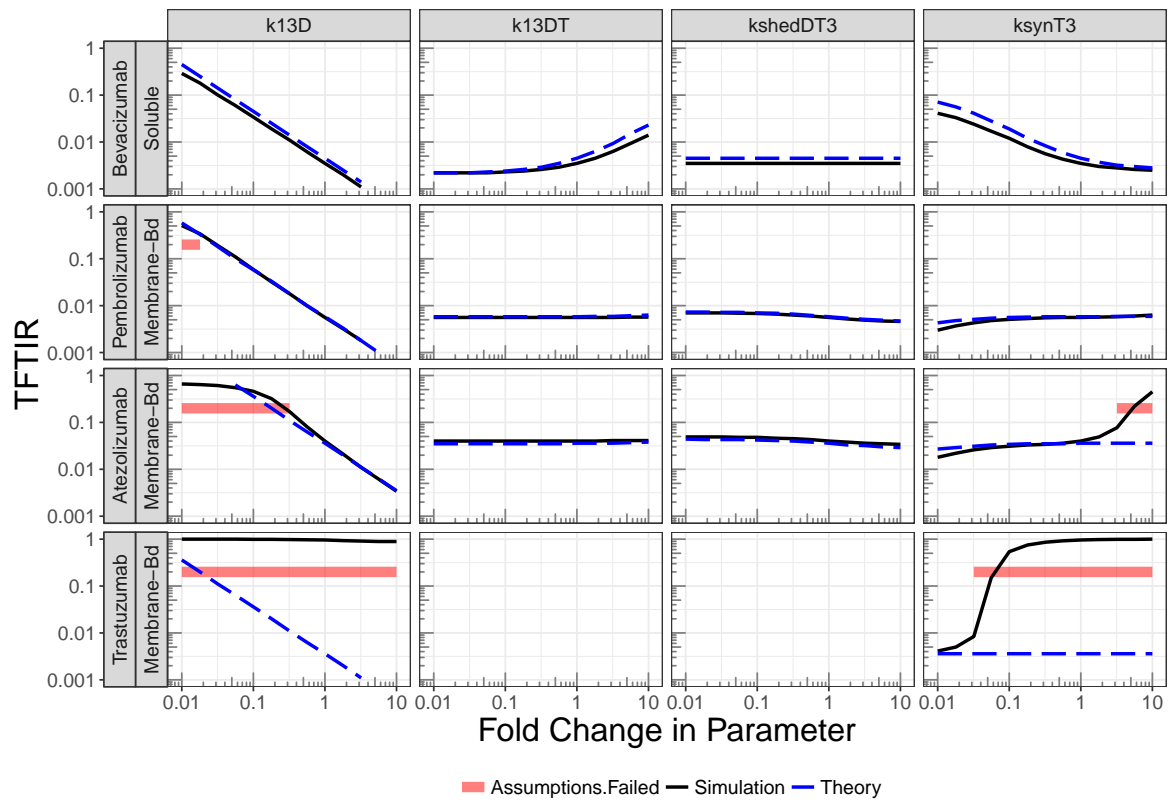

Fig. S4 Parameter exploration where the parameter at the top of each column is changed from $0.01 \times$ to $10 \times$ from its baseline value. There is in general good agreement between theory and experiment, except for trastuzumab, when the target expression is high. Kssd approximation. 
bioRxiv preprint doi: https://doi.org/10.1101/432500; this version posted February 6, 2019. The copyright holder for this preprint (which was not certified by peer review) is the author/funder, who has granted bioRxiv a license to display the preprint in perpetuity. It is made available under aCC-BY-NC 4.0 International license.

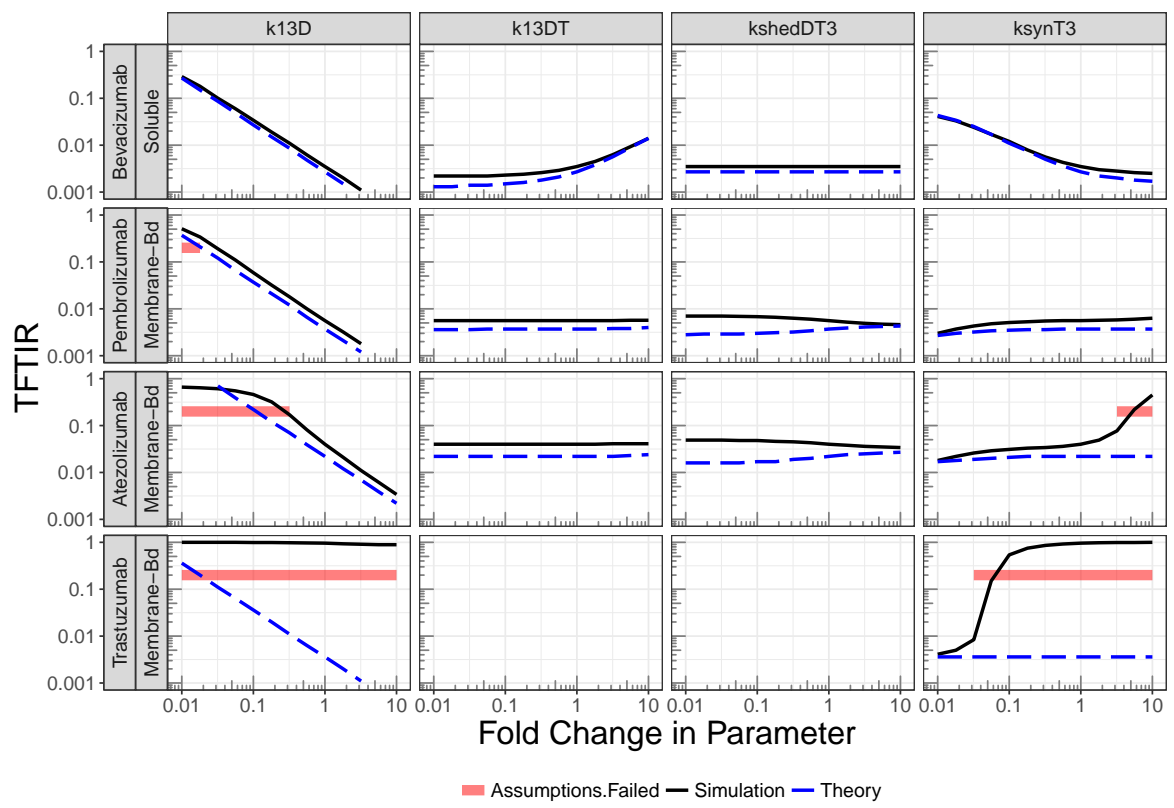

Fig. S5 Parameter exploration where the parameter at the top of each column is changed from $0.01 \times$ to $10 \times$ from its baseline value. There is in general good agreement between theory and experiment, except for trastuzumab, when the target expression is high. Kss approximation. 
bioRxiv preprint doi: https://doi. org/10.1101/432500; this version posted February 6, 2019. The copyright holder for this preprint (which was not certified by peer review) is the author/funder, who has granted bioRxiv a license to display the preprint in perpetuity. It is made available under aCC-BY-NC 4.0 International license.

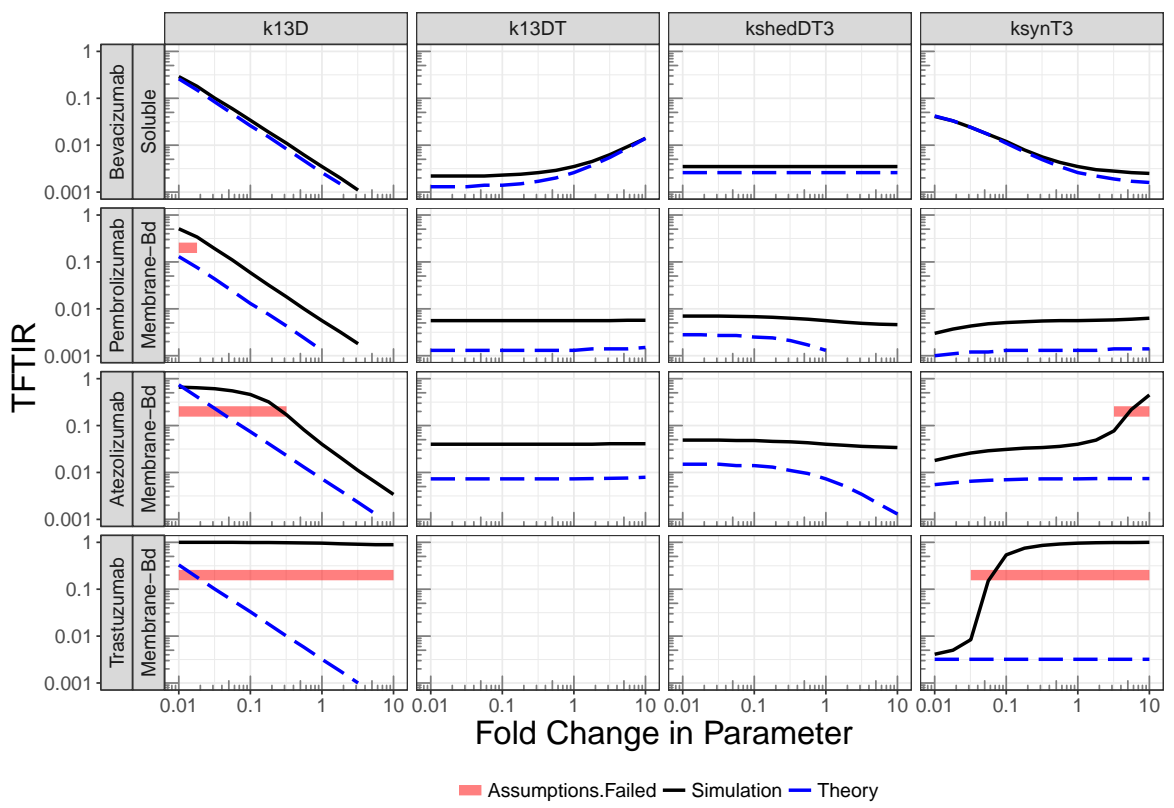

Fig. S6 Parameter exploration where the parameter at the top of each column is changed from $0.01 \times$ to $10 \times$ from its baseline value. There is in general good agreement between theory and experiment, except for trastuzumab, when the target expression is high. $\mathrm{Kd}$ approximation. 